\title{
On the local structure of spacetime in ghost-free bimetric theory and massive gravity
}

\author{
S.F. Hassan and Mikica Kocic \\ Department of Physics \& The Oskar Klein Centre, Stockholm University, \\ AlbaNova University Centre, SE-106 91 Stockholm, Sweden \\ E-mail: fawad@fysik.su.se, mikica.kocic@fysik.su.se
}

ABSTRACT: The ghost-free bimetric theory describes interactions of gravity with another spin-2 field in terms of two Lorentzian metrics. However, if the two metrics do not admit compatible notions of space and time, the formulation of the initial value problem becomes problematic. Furthermore, the interaction potential is given in terms of the square root of a matrix which is in general nonunique and possibly nonreal. In this paper we show that both these issues are evaded by requiring reality and general covariance of the equations. First we prove that the reality of the square root matrix leads to a classification of the allowed metrics in terms of the intersections of their null cones. Then, the requirement of general covariance further restricts the allowed metrics to geometries that admit compatible notions of space and time. It also selects a unique definition of the square root matrix. The restrictions are compatible with the equations of motion. These results ensure that the ghost-free bimetric theory can be defined unambiguously and that the two metrics always admit compatible $3+1$ decompositions, at least locally. In particular, these considerations rule out certain solutions of massive gravity with locally Closed Causal Curves, which have been used to argue that the theory is acausal.

Keywords: Classical Theories of Gravity, Cosmology of Theories beyond the SM

ARXIV EPRINT: 1706.07806 


\section{Contents}

1 Introduction and summary 1

1.1 Summary of results 3

1.2 The origin of square root matrix in spin-2 interactions 4

2 Theorem on real square roots and intersecting null cones $\quad 6$

2.1 Statement of the problem 6

2.2 Definition of causally coupled and null coupled metrics 8

2.3 Statement and proof of the theorem 9

2.3.1 Reality of the square root matrix 9

2.3.2 Canonical form for a pair of symmetric matrices 9

$\begin{array}{ll}2.3 .3 \text { Proof by enumeration } & 10\end{array}$

$\begin{array}{ll}2.4 & \text { Implications of the theorem } \\ \end{array}$

3 Unique choice of the square root matrix 13

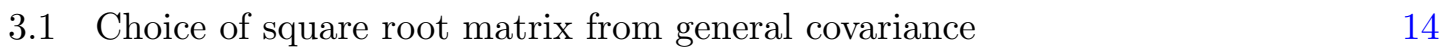

$\begin{array}{lll}3.2 & \text { Geometric significance of the principal root } & 15\end{array}$

$\begin{array}{ll}3.3 & \text { Type IV metrics as limits of Type IIb } \\ \end{array}$

$\begin{array}{ll}3.4 & \text { The absence of null coupled (Type IV) configurations } \\ \end{array}$

4 Remarks on causality and superluminality 19

$\begin{array}{lll}4.1 & \text { On the studies of acausality in massive gravity } 20\end{array}$

5 Discussion $\quad 22$

A The square root matrix function $\quad 24$

B Types of blocks in the theorem $\quad 25$

$\begin{array}{ll}\text { C Proof of the necessary condition } & 27\end{array}$

D Geometric mean of symmetric matrices $\quad 28$

$\begin{array}{llr}\text { E Absence of relative time orientation flip } & 29\end{array}$

\section{Introduction and summary}

Despite the overwhelming observational and theoretical evidence for physics beyond the standard model and general relativity, the absence of detailed observational data has so far hindered finding the underlying theory through the phenomenological model building. An alternative approach has been to investigate possible theoretical frameworks for the new physics guided by consistency requirements such as causal evolution and the absence of ghost instabilities, a well developed example being string theory. 
To recapitulate the situation from the low energy point of view, the standard model contains multiplets of spin- 0 , spin- $\frac{1}{2}$, and spin- 1 fields, and the multiplet structure (with the associated symmetries, broken or exact) are critical for the consistency and viability of the model. In contrast, general relativity is a theory of a single massless spin-2 field. The low energy limits of superstring theories and other grand unification schemes lead to similar field contents; characterized by a single massless spin-2 field, and a proliferation of lower spin multiplets. Hence, in the search for field theories beyond GR, a complementary approach would be to consider theories of gravity with extra spin-2 fields, in part motivated by the observation that many of the new physics signals are gravity related. Irrespective of their phenomenological relevance, it is an interesting theoretical question to find out if such theories could exist at all, or if GR is the unique (classically) consistent theory of a spin-2 field. This question has attracted considerable attention since the early works on massive $[1,2]$ and interacting spin-2 fields $[3],{ }^{1}$ but remains unresolved so far. ${ }^{2}$ The stumbling blocks to be avoided are instabilities, such as the Boulware-Deser (BD) ghost [2], and problems with causality due to nonminimal couplings of the metric. Ghost-free theories of massive gravity [6, 7] and interacting spin-2 fields [8,9] have now been constructed, but the complete classical consistency of these theories needs to be further investigated.

In this paper we consider the ghost-free bimetric theory [8] which is a theory of the gravitational metric $g_{\mu \nu}$, in the presence of an extra spin-2 field $f_{\mu \nu}$, and without the BD ghost. We address two potentially debilitating problems that are inherent in the construction of such theories and show that both are avoided by the natural requirements of the reality of the action and its general covariance. Our results also apply to ghost-free massive gravity $[6,7]$ which is obtainable from bimetric theory on freezing the dynamics of $f_{\mu \nu}$. However, in this case the implications are somewhat weaker. Here we briefly elaborate on the problems to be resolved and then present a brief summary of our results.

In formulating the ghost-free bimetric theory one encounters two potential problems that could render the theory ill-defined from the outset. One of the problems relates to the compatibility of the notions of space and time for the two metrics $g_{\mu \nu}$ and $f_{\mu \nu}$. Since each metric has an Einstein-Hilbert term in the action, as dictated by the absence of ghost, they come with their respective notions of space and time which, a priori, may not be compatible with each other. For example, if in any $3+1$ decomposition a common spacelike hypersurface does not exist, then formulating the dynamics of the theory as time evolution of initial data on a spatial hypersurface becomes problematic from the outset.

Another problem is that the interaction potential between the metrics $g_{\mu \nu}$ and $f_{\mu \nu}$ is given in terms of a matrix $S^{\mu}{ }_{\nu}$ defined through the equation $S^{2}=g^{-1} f$. The solutions are the matrix square roots $S=\sqrt{g^{-1} f}$, which are nonunique and possibly nonreal. To define the theory unambiguously, one must require $S$ to be real and also provide a criterion to specify it uniquely. Then one has to find the corresponding restrictions on $g_{\mu \nu}$ and $f_{\mu \nu}$ and ensure that they are consistent with the dynamics.

\footnotetext{
${ }^{1}$ The related work in [4] had remained completely unnoticed.

${ }^{2}$ Regarding multiple massless spin-2 fields, it has been shown in [5] that such theories with non-Abelian gauge symmetries cannot exist.
} 


\subsection{Summary of results}

The main result of this paper is a theorem showing that the reality condition on the equations leads to a classification of the allowed $g_{\mu \nu}$ and $f_{\mu \nu}$ configurations based on how their null cones intersect. Then compatibility with general covariance further restricts the allowed configurations to metrics $g_{\mu \nu}$ and $f_{\mu \nu}$ that admit compatible notions of space and time, consistent with the equations of motion. General covariance also leads to a unique definition of the square root matrix. This is elaborated below.

To ensure that the bimetric equations always yield real $g_{\mu \nu}$ and $f_{\mu \nu}$ as solutions, we require that only configurations for which the matrix $g^{-1} f$ admits real square roots are allowed. This requirement is converted into conditions on $g$ and $f$, and leads to four classes allowed metric configurations classified as Type I to Type IV (including some subtypes). Each metric Type has a geometric significance. In Type I-III configurations, the metrics $g_{\mu \nu}$ and $f_{\mu \nu}$ have null cones that always intersect such that they have common timelike and common spacelike directions, hence the metrics admit compatible notions of space and time. In Type IV, the null cones touch only along two null directions (the simplest example being $g=\operatorname{diag}(-1,1,1,1), f=\operatorname{diag}(1,-1,1,1))$ and the metrics do not admit compatible notions of space and time. In all other cases where the null cones do not intersect as above, $g^{-1} f$ does not admit any real square root.

For Type I-IV configurations the real square roots $S=\sqrt{g^{-1} f}$ are not unique. In particular, Type IV metrics only admit (an infinite number of) nonprimary real square roots. For nonprimary roots, $S^{\mu}{ }_{\nu}$ does not transform as a $(1,1)$ tensor and the theory will violate general covariance. To show that other configurations cannot dynamically evolve into Type IV, we note that, for Type IV metrics, $S$ coincides with branch cuts of the square root function and, around such configurations, the variation $\delta S$ is ill-defined. Hence such metrics are also excluded by the variational principle and the correct implementation of the equations of motion. We conclude that Type IV metrics are precluded by general covariance and this is consistent with the equations of motion.

For the remaining Type I-III configurations, in general, $g^{-1} f$ admits multiple real primary square roots. In particular, there is a unique principal root which is always primary and, hence, always consistent with general covariance. But the remaining nonprincipal roots can continuously degenerate into nonprimary roots in certain regions of the configuration space where $S^{\mu}{ }_{\nu}$ will not transform as a $(1,1)$ tensor. Hence, requiring that the theory must always respect general covariance for generic field configurations, implies that $S=\sqrt{g^{-1} f}$ must be defined as the unique real principal square root matrix which exists only for Type I-III metric configurations.

The above results ensure that (i) the ghost-free bimetric theory can be defined unambiguously in terms of the principal real square root matrix, and that (ii) the two metrics always admit compatible notions of spacetime and compatible $3+1$ decompositions, at least locally. However, it should be emphasized that this does not yet address the well-posedness of the initial value problem and causal evolution, which need to be further investigated.

Finally, we comment on the issues of superluminality and causality in bimetric theory and massive gravity. In particular, we point out that some analyses of acausality in massive 
gravity that indicate the presence of (local) closed causal curves involve configurations that are precluded by the definition of the theory described here.

The rest of this paper is organized as follows: in section 1.2 we review the origin of square root matrix in spin-2 interactions. In section 2 we state and prove the theorem on real square roots and interpret the resulting classification of allowed metrics in terms of the intersections of their null cones. The immediate implications for compatible $3+1$ decompositions are discussed in section 2.4. In section 3 we discuss the restrictions that lead to a unique definition of the square root matrix, as the principle root, based on general covariance. The geometric significance of the principal square root is explained in section 3.2. In section 4 we comment on causality and superluminality issues in general and then show that massive gravity backgrounds with local closed causal curves considered in the literature are disallowed configurations. Finally, section 5 is devoted to a brief discussion of our results. Many technical details are relegated to the appendices.

\subsection{The origin of square root matrix in spin-2 interactions}

On general grounds, a theory of the gravitational metric $g_{\mu \nu}$ interacting with another spin-2 field $f_{\mu \nu}$ has an action of the form,

$$
\mathcal{S}=\int \mathrm{d}^{4} x\left[m_{g}^{2} \sqrt{-g} R_{g}-m^{4} \sqrt{-g} V\left(g^{-1} f\right)+\mathcal{L}_{f}(\nabla f, f)\right]
$$

Here, $R_{g}$ is the curvature scalar of $g_{\mu \nu}$ and the potential $V$ can depend on the fields only through the matrix $g^{-1} f$ and its inverse, as required by general covariance. The Lagrangian $\mathcal{L}_{f}$ contains the kinetic terms for $f_{\mu \nu}$ and may also depend on $g_{\mu \nu}$. Dropping $\mathcal{L}_{f}$ and specifying $f_{\mu \nu}$ by hand, say, $f_{\mu \nu}=\eta_{\mu \nu}$, gives massive gravity in a fixed background. However, besides the five helicities of the massive spin-2 field $g_{\mu \nu}$, generally such a theory also propagates a Boulware-Deser (BD) ghost [2]. Similar arguments also imply the existence of a ghost in the theory with a dynamical $f_{\mu \nu}$. The challenge is to find a ghost-free combination of $V$ and $\mathcal{L}_{f}$.

The historical approach to the ghost problem, briefly described below, also sheds light on the structure of the ghost-free theory. In the massive gravity case, the choice $f_{\mu \nu}=\eta_{\mu \nu}$ breaks general covariance. The broken symmetry can be restored by the Stückelberg trick which amounts to replacing $\eta_{\mu \nu}$ by its coordinate transformation,

$$
\eta_{\mu \nu} \rightarrow \frac{\partial \phi^{a}}{\partial x^{\mu}} \eta_{a b} \frac{\partial \phi^{b}}{\partial x^{\nu}}
$$

The coordinate functions $\phi^{a}(x)$ are the Stückelberg fields. On decomposing these as $\phi^{a}=$ $x^{a}+\partial^{a} \pi+A^{a}$, and taking a certain flat space limit, refs. $[10,11]$ pointed out that a ghost in the original massive gravity implies an unhealthy theory for the scalar field $\pi(x)$. Namely, on setting $g_{\mu \nu}=\eta_{\mu \nu}$ and $A^{a}(x)=0$, the replacement (1.2) amounts to,

$$
\left(g^{-1} \eta\right)_{\nu}^{\mu} \rightarrow\left(\delta_{\lambda}^{\mu}+\partial^{\mu} \partial_{\lambda} \pi\right)\left(\delta_{\nu}^{\lambda}+\partial^{\lambda} \partial_{\nu} \pi\right)
$$


or, in an obvious matrix notation, $g^{-1} \eta \rightarrow(\mathbb{1}+\partial \partial \pi)^{2}$. Then $V\left(g^{-1} \eta\right)$ in (1.1) becomes a higher derivative Lagrangian for $\pi(x)$. If this has terms with more than two time derivatives, it will suffer from an Ostrogradsky instability (an unbounded nonpositive Hamiltonian) [12]. This instability was correlated to the BD ghost of the original nonlinear theory [10]. To find a ghost-free theory, the authors in [11] required that in the limit (1.3), $V$ should not produce terms with more than two time derivatives of $\pi(x)$ and, hence, the derivatives must appear in the antisymmetric combinations,

$$
V\left(g^{-1} \eta\right) \rightarrow \sum_{n=0}^{4} \alpha_{n} \epsilon_{\mu_{1} \cdots \mu_{n} \lambda_{n+1} \cdots \lambda_{4}} \epsilon^{\nu_{1} \cdots \nu_{n} \lambda_{n+1} \cdots \lambda_{4}} \partial^{\mu_{1}} \partial_{\nu_{1}} \pi \cdots \partial^{\mu_{n}} \partial_{\nu_{n}} \pi .
$$

This is a necessary condition for the absence of ghost in massive gravity. It is easy to see that this condition also uniquely determines the nonlinear form of $V\left(g^{-1} \eta\right)$ since (1.3) implies the reverse replacement,

$$
\delta_{\nu}^{\mu}+\partial^{\mu} \partial_{\nu} \pi \rightarrow\left(\sqrt{g^{-1} \eta}\right)_{\nu}^{\mu} .
$$

Then, eliminating the $\partial^{\mu} \partial_{\nu} \pi$ factors through this replacement, gives the desired potential. This was implemented in [6], based on an alternative analysis of [13], which also verified the absence of the ghost at the next order. A compact general expression, based directly on (1.4) and valid for any $f_{\mu \nu}$ is [14],

$$
V\left(\sqrt{g^{-1} f}\right)=\sum_{n=0}^{4} \beta_{n} e_{n}\left(\sqrt{g^{-1} f}\right) .
$$

The parameters $\beta_{n}$ are linear combinations of the $\alpha_{n}$ in (1.4), and $e_{n}(S)$ are the elementary symmetric polynomials of the eigenvalues of the matrix $S$ whose explicit forms are not needed here. Note that the potential is uniquely determined by the necessary condition (1.4) of [11] for the absence of ghost in a specific limit. Although the reasoning is valid for $f_{\mu \nu}=\eta_{\mu \nu}$, we keep $f_{\mu \nu}$ general. An unusual feature of $V$ is that it depends on the square root of the matrix $g^{-1} f$, which is, in general, nonunique and not necessarily real. ${ }^{3}$ Given the potential $V$, one can show the absence of the BD ghost in the fully nonlinear theory $[7,9,15,16]$. Further work on the ghost problem in massive gravity can be found in $[17-20]$.

Finally, the field $f_{\mu \nu}$ can be rendered dynamical without reintroducing the BD ghost only through an Einstein-Hilbert action. The ghost-free theory of two interacting spin-2 fields is then given by [8],

$$
\mathcal{S}=\int \mathrm{d}^{4} x\left[m_{g}^{2} \sqrt{-g} R_{g}-m^{4} \sqrt{-g} V\left(\sqrt{g^{-1} f}\right)+m_{f}^{2} \sqrt{-f} R_{f}\right] .
$$

A Hamiltonian analysis $[8,9]$ shows that this theory propagates seven modes which decompose into a massive and a massless spin-2 fluctuation around Einstein backgrounds [22].

\footnotetext{
${ }^{3}$ The reasoning that leads to $(1.5)$ assumes $\sqrt{(\mathbb{1}+\partial \partial \pi)^{2}}=\mathbb{1}+\partial \partial \pi$, analogous to $\sqrt{x^{2}}=x$, whereas, on specifying a branch, $\sqrt{x^{2}}= \pm|x|$. The difference can be ignored for small $\partial \partial \pi$. Early works also use a binomial expansion for $(\mathbb{1}+h)^{1 / 2}$, discussed in section 5 .
} 
However, the ghost analysis in $[7-9,15,16]$ relies on the assumption that the two metrics admit simultaneous $3+1$ decompositions in terms of lapse and shift variables. The complete validity of this assumption follows from the results of the present paper. For a review of the subsequent developments in the field, see [23, 24].

The $g$ and $f$ metrics can be minimally coupled (as in GR) to two different types of matter. As long as these matter types do not interact directly with each other, the theory remains ghost-free. If we identify $g_{\mu \nu}$ as the gravitational metric, then $m_{f} / m_{g} \rightarrow \infty$ is the massive gravity limit $[8,25]$, while $m_{f} / m_{g} \rightarrow 0$ is the General Relativity limit [26].

\section{Theorem on real square roots and intersecting null cones}

In this section we first outline two potential problems that could render bimetric theory ill-defined, and then prove a theorem that addresses both issues. The implications are discussed in the following sections.

\subsection{Statement of the problem}

The previous section emphasized two main features of the ghost-free bimetric theory (1.7), namely, its dependence on the square root matrix $S=\sqrt{g^{-1} f}$, and that both metrics $g_{\mu \nu}$ and $f_{\mu \nu}$ have Einstein-Hilbert terms in the action. These lead to two potential problems:

1. A square root matrix function $S$ defined through $S^{2}=g^{-1} f$ is not unique and not necessarily real. It can be primary or nonprimary, furthermore, primary square roots have multiple branches (as many as 16 for a $4 \times 4$ matrix), and the nonprimary ones are infinite in number (appendix A contains an overview of matrix square roots). Thus, without a rule for dealing with the square root, the theory is ambiguously specified. To avoid this, the definition of the theory must include a requirement that $g^{-1} f$ has real square roots, and also a prescription for selecting one of the roots (similar to requiring invertibility of $g_{\mu \nu}$ ). Then the question is what restrictions this choice of $S$ imposes on $g$ and $f .{ }^{4}$

2. By definition, $g_{\mu \nu}$ and $f_{\mu \nu}$ are Lorentzian metrics of signature $(1,3)$. In general, two such metrics may not admit simultaneous proper $3+1$ decompositions even locally, that is, they may not admit compatible notions of space and time, leading to potential inconsistencies outlined below.

Recall that in General Relativity (GR) there always exist local coordinate systems in which the metric $g_{\mu \nu}$ admits a proper $3+1$ decomposition, that is, with a real lapse function $N$ and a positive definite spatial metric $g_{i j}$ (see, for example, [28]). This provides the spacelike hypersurfaces on which initial data can be specified for local evolution. On restricting to globally hyperbolic spacetimes, such $3+1$ decompositions can be extended

\footnotetext{
${ }^{4}$ In specific cases one may be able to make sense of nonreal square roots, for example allowing imaginary $S$ (corresponding to $f=-c^{2} g$ ) when the action contains only even powers of $S$ [27]. But such procedures do not generalize to arbitrary $g$ and $f$ configurations that may result in nonreal $S$. Hence such possibilities are not considered here.
} 


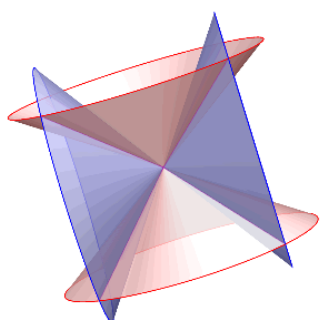

(a)

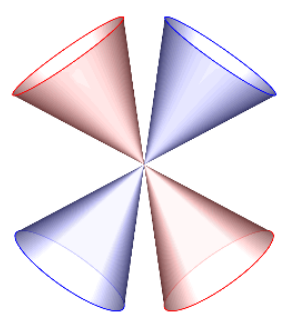

(b)

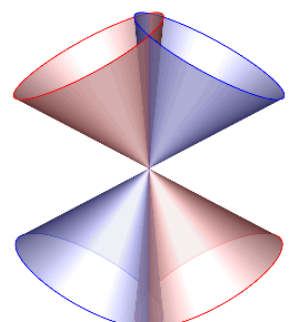

(c)

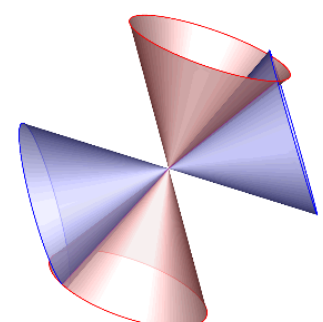

(d)

Figure 1. Some possible intersections of two null cones.

globally $[29,30]$. Also, the Einstein-Hilbert action naturally splits $g_{\mu \nu}$ into dynamical $g_{i j}$ and the nondynamical $N, N_{i}$ variables, consistent with the $3+1$ decomposition. The existence of a global time allows for a Hamiltonian formulation of the theory [31, 32].

The two Einstein-Hilbert terms in the bimetric action (1.7) indicate that, for each metric, a $3+1$ decomposition is still relevant for isolating its dynamical content. The absence of compatible $3+1$ splits for the two metrics then leads to inconsistent evolution equations, even locally. For example, when such metrics couple to their respective matter sectors, there may not exist common hypersurfaces for specifying initial data for both sectors. Hence, consistent time evolution requires that the metrics admit compatible $3+1$ decompositions (although this alone is not enough for the Cauchy problem to be wellposed). Note that in massive gravity, where the $f$ metric is nondynamical, the existence of compatible $3+1$ decompositions is not a requirement, unless the theory is regarded as a bimetric limit.

The situation is easily illustrated in terms of the null cones of the two metrics, with a few examples shown in figure 1 (a longer list is given later). ${ }^{5}$

The null cones in case (a) have common timelike directions within their intersection, but no common spacelike hypersurfaces, hence they do not admit simultaneous proper $3+1$ decompositions. In cases (b) and (c), the two metrics admit compatible $3+1$ decompositions with real lapses and common spatial hypersurfaces. However, in case (b) the choice of time and space directions is not unique as the time axis can be chosen either vertically or horizontally. As a result, the notion of time orientation is not unique (an upward time axis aligns with the future of $g$ and $f$ cones, whereas a horizontal time axis could align with the future of $g$ and past of $f$ ). Only in case (c) the null cones are interlocked along common timelike directions within their intersection and have the same relative time orientation. Case (d) is a coordinate transformation of (c) and admits proper $3+1$ splits for correctly chosen spatial hypersurfaces. In the fol-

\footnotetext{
${ }^{5}$ We use the term of null cone instead of light cone. To be specific, at a point $p$ on a spacetime manifold $M$, a null cone is a subset of the tangent space at $p \in M$. Relative to some metric, it is a set of all null vectors at $p$, excluding the zero vector. The interior of a null cone is a set of all timelike vectors while its exterior is a set of all spacelike vectors at $p$. A surface element through $p$ is called spacelike if and only if its normal is timelike, and null if and only if its normal is null. A spacelike surface is one which is spacelike everywhere. A spacelike surface element through $p$ does not intersect the null cone, i.e., it contains only the zero vector and separates the two parts of the null cone into past and future.
} 

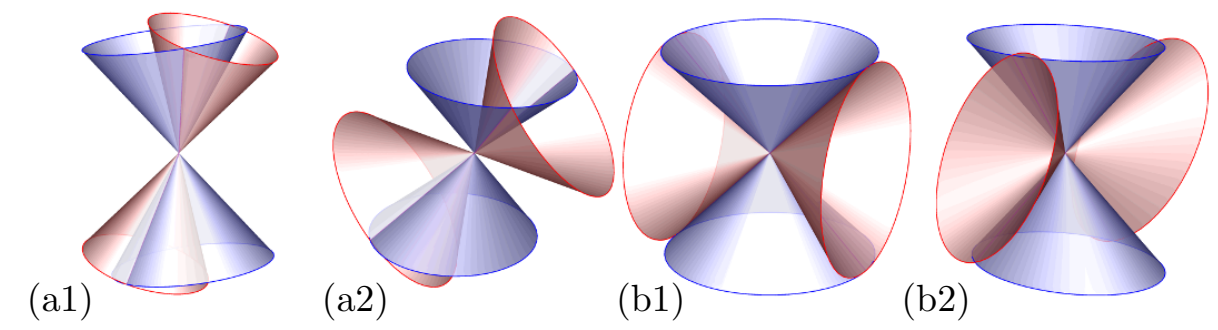

Figure 2. (a) Causally coupled, and (b) Null coupled cones.

(a)

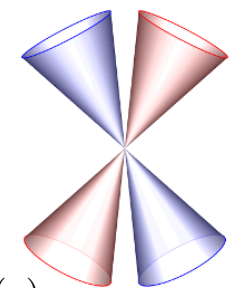

(b)

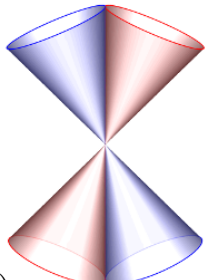

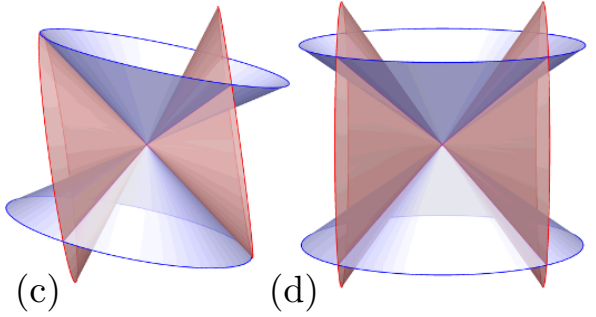

(d)

lowing it is enough to consider metrics up to diffeomorphisms, so cases (c) and (d) are not distinguished. ${ }^{6}$

\subsection{Definition of causally coupled and null coupled metrics}

We introduce some terminology to distinguish two types of null cone intersections. Let $M$ be a manifold equipped with two arbitrary Lorentzian metric tensors $f$ and $g$, each of signature $(1, n)$. Consider their null cones at some point $p \in M$. We say that $f$ and $g$ (or their null cones) are causally coupled at $p$ if and only if there exists, through $p$, a common timelike vector and a common spacelike surface element relative to both $f$ and g. Examples are shown in figure 2(a). We say that $f$ and $g$ (or their null cones) are null coupled at $p$ if and only if there neither exists a common timelike vector nor a common spacelike surface element relative to both $f$ and $g$ at $p$, i.e., when the null cones touch along two null directions. Examples are shown in figure 2(b).

For comparison, figure 3 shows typical cases when $f$ and $g$ are neither causally coupled nor null coupled. The above definitions are local statements about the topology of null cones, independent of the coordinate system. Recall that the interior (and the exterior) of a null cone is an open set, and that the intersection of two open sets is an open set. When the $f$ and $g$ null cones are causally coupled, their interiors and exteriors intersect so that one can always find an inner cone inside the intersection of their interiors. Similarly one can always find an outer cone the interior of which contains the null cones of both $f$ and g. A special case is when one null cone is contained within the other.

\footnotetext{
${ }^{6}$ Besides the tangent space null cones, given by $g_{\mu \nu} v^{\mu} v^{\nu}=0$, one may also consider cotangent, or momentum space null cones, $g^{\mu \nu} k_{\mu} k_{\nu}=0$. The momentum space null cones corresponding to figure 1(a) have the topology of case (b) and vice versa. Only in the intersecting case (c), the tangent and the cotangent space null cones have similar topology.
} 


\subsection{Statement and proof of the theorem}

We now state the main result of this paper as a theorem and provide a proof.

Theorem. Let $f$ and $g$ be two regular Lorentzian metric tensors. A real square root of $A \equiv g^{-1} f$ exists if and only if $f$ and $g$ are either causally coupled or null coupled.

As described in appendix A, a matrix can have several real primary square roots and an infinite number of nonprimary roots. The theorem applies regardless of which one of these is chosen, but it also provides a classification of the real roots. Then, as explained in the next section, the requirement of general covariance, leads to a unique choice for the square root.

Here we prove that the reality of $\sqrt{g^{-1} f}$ is sufficient for $f$ and $g$ to be causally coupled or null coupled (this is the "only if" part of the theorem). The proof of the necessary condition is less instructive and is relegated to appendix C. The strategy of the sufficiency proof is first to state the conditions under which $A$ admits real square roots and then, for $A=g^{-1} f$, to find the corresponding restrictions on $g$ and $f$ using a theorem on the canonical form of a pair of matrices. We work locally at some point $p$ of a manifold of finite dimension $d=1+n$, with 1 time and $n \geq 1$ space dimensions. $f_{\mu \nu}$ and $g_{\mu \nu}$ are nonsingular real symmetric tensors. Locally, congruences $Z^{\top} g Z$ and $Z^{\top} f Z$, with real $Z$, are equivalent to coordinate transformations at $p$.

\subsubsection{Reality of the square root matrix}

All eigenvalues of $g$ and $f$ are real, but the matrix $A=g^{-1} f$ is not symmetric and can have complex eigenvalues. Let us denote its real eigenvalues by $\lambda_{k}, 1 \leq k \leq q$, and the complex ones, which come in conjugate pairs, by $a_{k} \pm \mathrm{i} b_{k}, k \geq q+1$. A may not be diagonalizable, but it always has a Jordan normal form (see section 2.3.2). A real square root $\sqrt{A}$ exists only in the following two cases (see theorems 1.23, 1.26 and 1.29 in [33], see also [34, 35]):

1. No eigenvalue of $A$ lies on the negative real axis $\mathbb{R}^{-}$. This restricts real eigenvalues to $\lambda_{k}>0,1 \leq k \leq q$, but there is no restriction on complex eigenvalues.

2. The matrix $A$ has real negative eigenvalues $\lambda_{k}<0$, but each such eigenvalue appears in an even number of Jordan blocks of the same size.

$A$ can be put in Jordan normal form, $A=Z A_{\mathrm{J}} Z^{-1}$, by some appropriate transformation $Z$. Then, by definition, $\sqrt{A}=Z \sqrt{A_{\mathrm{J}}} Z^{-1}$, where the square roots $\sqrt{A_{\mathrm{J}}}$ can be explicitly computed by standard methods (see appendix A). $\sqrt{A}$ is a real matrix only in the above two cases even when it has complex eigenvalues, hence the action and the equations of motion will be real. Below we convert the above conditions on $A$ into restrictions on $g$ and $f$ and express them in terms of null cone intersections.

\subsubsection{Canonical form for a pair of symmetric matrices}

The transformation $Z$ that converts $A$ to its Jordan normal form $A=Z A_{\mathrm{J}} Z^{-1}$ is not unique. For $A=g^{-1} f$, the arbitrariness in $Z$ can be used to put $g$ and $f$ in a specific form, $Z^{\top} g Z, Z^{\top} f Z$, determined by the structure of $A_{\mathrm{J}}$. This is accomplished by a theorem 
on canonical pair form, a proof of which can be found in [36]. ${ }^{7}$ Then, conditions on the Jordan normal form of $g^{-1} f$ can be converted into restrictions on $g$ and $f$.

The theorem on canonical pair form states that, given nonsingular real symmetric matrices $g$ and $f$, there always exists a real, nonsingular transformation $Z$ that converts $g^{-1} f$ to its Jordan normal form and, at the same time, puts $g$ and $f$ in specific forms,

$$
\begin{aligned}
Z^{-1} g^{-1} f Z & =\operatorname{diag}\left(J_{1}, \ldots, J_{q}, C_{q+1}, \ldots, C_{p}\right), \\
Z^{\top} g Z & =\operatorname{diag}\left(\epsilon_{1} E_{1}, \ldots, \epsilon_{q} E_{q}, E_{q+1}, \ldots, E_{p}\right), \\
Z^{\top} f Z & =\operatorname{diag}\left(\epsilon_{1} E_{1} J_{1}, \ldots, \epsilon_{q} E_{q} J_{q}, E_{q+1} C_{q+1}, \ldots, E_{p} C_{p}\right) .
\end{aligned}
$$

The first line is simply the Jordan normal form of $g^{-1} f$ where $J_{k}$ are the $n_{k} \times n_{k}$ Jordan blocks (for $1 \leq k \leq q$ ) corresponding to the real eigenvalues $\lambda_{k}$ of $A$, while $C_{k}$ are the $n_{k} \times n_{k}$ real Jordan blocks (for $k \geq q+1$ and even $n_{k}$ ) corresponding to the pairs of complex conjugate eigenvalues $a_{k} \pm \mathrm{i} b_{k}$ of $A$,

$$
J_{k} \equiv\left(\begin{array}{ccccc}
\lambda_{k} & 1 & & \\
& \lambda_{k} & \ddots & \\
& & \ddots & 1 \\
& & & \lambda_{k}
\end{array}\right), \quad C_{k} \equiv\left(\begin{array}{cccc}
\Lambda_{k} & I_{2} & & \\
& \Lambda_{k} & \ddots & \\
& & \ddots & I_{2} \\
& & & \Lambda_{k}
\end{array}\right), \Lambda_{k} \equiv\left(\begin{array}{cc}
a_{k} & -b_{k} \\
b_{k} & a_{k}
\end{array}\right), I_{2} \equiv\left(\begin{array}{ll}
1 & 0 \\
0 & 1
\end{array}\right)
$$

The transformation $Z$ in (2.1) is not uniquely fixed since there are many matrices that commute with Jordan form on the right hand side. The freedom in choosing $Z$ is just enough to transform $g$ as in equation (2.2), where $\epsilon_{k} \in\{ \pm 1\}$ are possible signs related to the signature of $g$, and $E_{k}=E_{k}^{-1}$ is the $n_{k} \times n_{k}$ exchange (or reversal) matrix,

$$
E_{k} \equiv\left({ }_{1} \cdot{ }^{1}\right)
$$

Here, $\operatorname{dim} E_{k}=\operatorname{dim} J_{k}$ for $k \leq q$ and $\operatorname{dim} E_{k}=\operatorname{dim} C_{k}$ for $k>q$. Finally, given $g^{-1} f$ and $g, f$ can be computed to be (2.3). While all eigenvalues of $f$ and $g$ are necessarily real, this may not hold for $g^{-1} f$. Nonetheless, all matrices in the theorem are real, including $Z$.

We again emphasize that, at any point $p$, the congruence or similarity transformation $Z$ can be interpreted as a local coordinate transformation. In fact, since $Z^{\top} g Z$ in (2.2) is the Lorentz frame metric $\eta$, at most in a nondiagonal form, $Z$ coincides with the vielbein of the metric $g$ in such a Lorentz basis.

\subsubsection{Proof by enumeration}

Using the above decomposition, we now enumerate all possible types of matrices $g$ and $f$ subject to the conditions that the real eigenvalues $\lambda_{k}$ of $g^{-1} f$ are restricted by the reality

\footnotetext{
${ }^{7}$ Many authors, starting with Weierstrass and Kronecker, have worked on the question of canonical forms for a pair of real symmetric matrices. For a historical and mathematical survey see [37, 38], also [39-41].
} 
of $\sqrt{g^{-1} f}$, as specified in subsection 2.3.1, while the complex eigenvalues are unrestricted. Also the following obvious restrictions apply:

1. Let $\rho^{-}\left(\rho^{+}\right)$denote the number of negative (positive) eigenvalues of a matrix. Then,

$$
\rho^{-}(g)=\rho^{-}(f)=1, \quad \rho^{+}(f)=\rho^{+}(g)=n, \quad d=n+1 \geq 2 .
$$

2. The number and type of Jordan blocks may vary, yet they always satisfy $\operatorname{dim} E_{k}=$ $\operatorname{dim} J_{k}$ for $k \leq q$, corresponding to real eigenvalues $\lambda_{k}$, and $\operatorname{dim} E_{k}=\operatorname{dim} C_{k}$ for $k>q$, corresponding to the complex eigenvalues $a_{k} \pm \mathrm{i} b_{k}$.

The signature of $g$, decomposed as in (2.2), imposes the following condition on the $E_{k}$ 's,

$$
1=\rho^{-}(g)=\sum_{1 \leq k \leq q} \rho^{-}\left(\epsilon_{k} E_{k}\right)+\sum_{q<k \leq p} \rho^{-}\left(E_{k}\right),
$$

where, for the matrices $E_{k}$ given by (2.5) one has,

$$
\rho^{-}\left(E_{k}\right)=\left\lfloor\frac{1}{2} n_{k}\right\rfloor, \quad \rho^{+}\left(E_{k}\right)=\left\lfloor\frac{1}{2}\left(n_{k}+1\right)\right\rfloor, \quad n_{k}=\operatorname{dim} E_{k} .
$$

Thus, up to permutations of the blocks, $Z^{\top} g Z$ can only have a finite number of possible forms which restrict $Z^{\top} f Z$ accordingly, given the Jordan form of $g^{-1} f$. Denoting $Z^{\top} g Z$ and $Z^{\top} f Z$ as $g$ and $f$ for brevity, all the possibilities are enumerated below. Here, we focus on $3+1$ dimensions for definiteness, but the results easily generalize to $n+1$ dimensions.

No $\boldsymbol{E}_{\boldsymbol{k}}$ with $\boldsymbol{n}_{\boldsymbol{k}}>\mathbf{1}$. Here, $g=\operatorname{diag}\left(\epsilon_{1}, \epsilon_{2}, \epsilon_{3}, \epsilon_{4}\right)$ has only one negative $\epsilon_{k}$, which we can assume to be $\epsilon_{1}=-1$. Then, $\operatorname{dim} J_{k}=1$ implies $g^{-1} f=\operatorname{diag}\left(\lambda_{1}, \lambda_{2}, \lambda_{3}, \lambda_{4}\right)$ and $f=\operatorname{diag}\left(-\lambda_{1}, \lambda_{2}, \lambda_{3}, \lambda_{4}\right)$, with real $\lambda_{k}$. The reality of the square root requires the following: (i) Either all $\lambda_{k}>0$, or, (ii) there exists one pair of negative eigenvalues $\lambda_{i}=\lambda_{j}<0$ with other $\lambda_{k}>0$, or (iii) more than one pair of type $\lambda_{i}=\lambda_{j}<0$, and other $\lambda_{k}>0$. Finally, $\rho^{-}(f)=1$ allows at most one negative pair ruling out (iii) and also requires the negative pair to include $\lambda_{1}$. Thus only two types of configurations are allowed (with $\lambda, \lambda_{k}>0$ ),

$$
\begin{array}{lll}
\text { Type I : } & g=\operatorname{diag}(-1,1,1,1), & f=\operatorname{diag}\left(-\lambda_{1}, \lambda_{2}, \lambda_{3}, \lambda_{4}\right), \\
\text { Type IV : } & g=\operatorname{diag}(-1,1,1,1), & f=\operatorname{diag}\left(\lambda,-\lambda, \lambda_{3}, \lambda_{4}\right) .
\end{array}
$$

One $\boldsymbol{E}_{\boldsymbol{k}}$ with $\boldsymbol{n}_{\boldsymbol{k}}=\mathbf{2}$. In this case, $g=\operatorname{diag}\left(\epsilon_{1} E_{1}, 1,1\right)$ with $\operatorname{dim} E_{1}=2$. When $\epsilon_{1}=+1$, $g^{-1} f$ contains either a $2 \times 2 J_{1}$ block or a $2 \times 2 C_{1}$ block, with the remaining $J_{k}$ being one dimensional. Then the reality of the square root and $\rho^{-}(f)=1$ leads to $\left(\lambda, \lambda_{k}>0, b \neq 0\right)$,

$$
\begin{array}{ll}
\text { Type IIa : } & g=\operatorname{diag}\left(\left(\begin{array}{ll}
0 & 1 \\
1 & 0
\end{array}\right), 1,1\right), \quad f=\operatorname{diag}\left(\left(\begin{array}{ll}
0 & \lambda \\
\lambda & 1
\end{array}\right), \lambda_{2}, \lambda_{3}\right), \\
\text { Type IIb : } & g=\operatorname{diag}\left(\left(\begin{array}{ll}
0 & 1 \\
1 & 0
\end{array}\right), 1,1\right), \quad f=\operatorname{diag}\left(\left(\begin{array}{rr}
b & a \\
a & -b
\end{array}\right), \lambda_{2}, \lambda_{3}\right) .
\end{array}
$$

The case with $\epsilon_{1}=-1$ corresponds to Type IIa with the sign of the $2 \times 2$ block reversed. When $\epsilon_{1}=1$ the $f$ null cone is inside the $g$ null cone and vice versa for $\epsilon_{1}=-1$. But except for the interchange of the metrics, the topology of the intersection remains unchanged. 


\begin{tabular}{|c|c|c|c|c|}
\hline Type & Segre char. & $\operatorname{diag}(g)$ & $\operatorname{diag}(f)$ & $\operatorname{diag}\left(g^{-1} f\right)$ \\
\hline $\mathbf{I}$ & [1111] & $(-1,1,1,1)$ & $\left(-\lambda_{1}, \lambda_{2}, \lambda_{3}, \lambda_{4}\right)$ & $\left(\lambda_{1}, \lambda_{2}, \lambda_{3}, \lambda_{4}\right)$ \\
\hline IIa & [211] & $\left( \pm\left(\begin{array}{ll}0 & 1 \\
1 & 0\end{array}\right), 1,1\right)$ & $\left( \pm\left(\begin{array}{ll}0 & \lambda \\
\lambda & 1\end{array}\right), \lambda_{2}, \lambda_{3}\right)$ & $\left(\left(\begin{array}{ll}\lambda & 1 \\
0 & \lambda\end{array}\right), \lambda_{2}, \lambda_{3}\right)$ \\
\hline IIb & {$[z \bar{z} 11]$} & $\left( \pm\left(\begin{array}{ll}0 & 1 \\
1 & 0\end{array}\right), 1,1\right)$ & $\left( \pm\left(\begin{array}{rr}b & a \\
a & -b\end{array}\right), \lambda_{2}, \lambda_{3}\right)$ & $\left(\left(\begin{array}{rr}a & -b \\
b & a\end{array}\right), \lambda_{2}, \lambda_{3}\right)$ \\
\hline III & {$[31]$} & $\left(\left(\begin{array}{lll}0 & 0 & 1 \\
0 & 1 & 0 \\
1 & 0 & 0\end{array}\right), 1\right)$ & $\left(\left(\begin{array}{lll}0 & 0 & \lambda \\
0 & \lambda & 1 \\
\lambda & 1 & 0\end{array}\right), \lambda_{2}\right)$ & $\left(\left(\begin{array}{lll}\lambda & 1 & 0 \\
0 & \lambda & 1 \\
0 & 0 & \lambda\end{array}\right), \lambda_{2}\right)$ \\
\hline IV & [(11)11] & $(-1,1,1,1)$ & $\left(\lambda,-\lambda, \lambda_{2}, \lambda_{3}\right)$ & $\left(-\lambda,-\lambda, \lambda_{2}, \lambda_{3}\right)$ \\
\hline
\end{tabular}

Table 1. Overview of all possible metric configurations for $d=4$.

One $\boldsymbol{E}_{\boldsymbol{k}}$ with $\boldsymbol{n}_{\boldsymbol{k}}=\mathbf{3}$. Here, the only allowed configuration is with $3 \times 3$ blocks $E_{1}, J_{1}$,

$$
\text { Type III : } \quad g=\operatorname{diag}\left(E_{1}, 1\right), \quad f=\operatorname{diag}\left(E_{1} J_{1}, \lambda_{2}\right) .
$$

In all the above cases $g^{-1}$ has the same matrix as $g$; hence, reintroducing the factors of $Z$, the matrix of $Z^{-1} g^{-1} f Z$ can be easily computed. The enumeration (2.9)-(2.12) is valid for all spatial dimensions $n \geq 1$, while the presence of Type III is restricted to $n \geq 2 .{ }^{8}$ Finally, the explicit construction of a common timelike vector and a common spacelike surface element for each Type is given in appendix B, completing the sufficiency proof. A comprehensive overview of the allowed configurations is given in table 1 with the Segre characteristics of $g^{-1} f$ shown in the second column. ${ }^{9}$

The possible configurations of the metrics are easily visualized in terms of the intersections of their null cones. The $Z$ transformation deforms the cones, but does not change the nature of their intersections. For each Type, the null cones are depicted, by example, in figure 4 in a convenient coordinate system.

From figure 4 it is obvious that for the configuration Types I-III, the null cones are causally coupled, while in Type IV, they are null coupled (using the terminology of section 2.2). The permitted configuration types I-IV correspond to the null cone intersections depicted in figure 4 for all allowed values of the parameters $\lambda, \lambda_{k}, a, b$. This is easy to verify analytically using the equations in appendix B, or simply by plotting the null cones for different parameter values.

Of the allowed configurations in 4 dimensions, only Type I and Type IIb can involve the full set of 20 components in $f$ and $g$, with 16 components in $Z$ and 4 components in $Z^{\top} f Z$. But the maximum number of independent field components is 18 for Type III,

\footnotetext{
${ }^{8}$ In dimensions higher than $d=2$, only a common positive definite part of the two metrics is affected by the additional positive eigenvalues.

${ }^{9}$ The Segre characteristic is a descending list of integers that correspond to the sizes of the blocks in a Jordan normal form where complex blocks are designated by $z \bar{z}$ instead. The integers representing submatrices with the same eigenvalue are listed together in parentheses; for example, [(21)1] denotes a derogatory Type IIa, [211], where $\lambda=\lambda_{2} \neq \lambda_{3}$.
} 


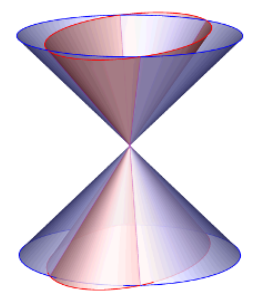

Type I

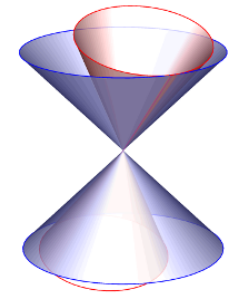

Type IIa

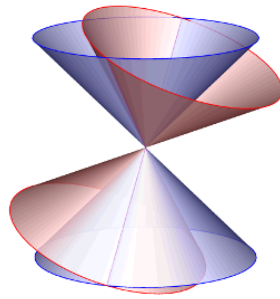

Type IIb

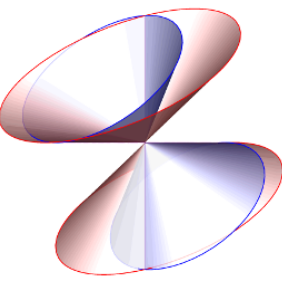

Type III

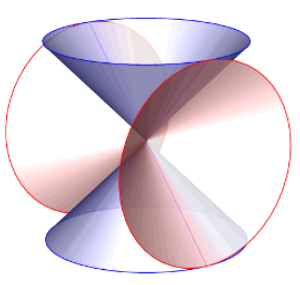

Type IV

Figure 4. Null cones corresponding to different allowed $g$ and $f$ metrics.

and 19 for Type IIa and Type IV. In section 3 we show that Type IV is an isolated class. However the other configurations are not isolated as discussed in the last paragraph of section 3.4. Explicit examples of the allowed configurations are the two metric ansätze considered in [42], for which null cones belong to subsets of Type IIa and Type I.

\subsection{Implications of the theorem}

The immediate implication of the above theorem is that the reality of bimetric equations of motion restricts the metrics to be either causally coupled (Type I-III) or null coupled (Type IV), as depicted in figure 4. Causally coupled metrics admit simultaneous proper $3+1$ decompositions, hence, the equations of motion can be recast as time evolution equations, as a first step towards formulating the Cauchy problem. The interlocking of the null cones ensures that if the spacetime manifold is time-orientable with respect to the metric $g$, then it is also time-orientable with respect to $f$ and vice versa.

Null coupled (Type IV) metrics do not admit simultaneous proper $3+1$ decompositions and would be problematic if allowed by the theory. Some of these issues will be discussed later. The simplest realization of such configurations is $g=\operatorname{diag}(-1,1,1,1)$, $f=\operatorname{diag}(1,-1,1,1)$. In general relativity, the metric also admits a d'Inverno-StachelSmallwood (DSS) 2+2 decomposition [43, 44], and initial data can be specified on a pair of null hypersurfaces. Since null coupled metrics share two null hyperplanes, they admit simultaneous $2+2$ decompositions but still remain problematic. The next section shows that Type IV configurations can and should be consistently discarded.

Metrics that are neither causally coupled nor null coupled, as in figure 3, result in complex equations of motion and are disallowed. If such configurations occurred, timeorientability with respect to one metric would not ensure the same for the other metric. Note that, although the configuration in figure 3(b) seems to be a limit of the causally coupled Type IIb cones in figure 4, with the intersection shrunk to zero, in reality, it is not obtainable from any configuration with real $\sqrt{g^{-1} f}$. Instead, it arises from metrics parameterized as Type IIa in (2.11), but with $\lambda<0$, corresponding to a nonreal $\sqrt{g^{-1} f}$.

\section{Unique choice of the square root matrix}

The reality condition alone does not determine square root matrix $S=\sqrt{g^{-1} f}$ uniquely. In this section we show that general covariance uniquely restrict $S$ to the primary square root 
on the principal branch, ruling out, in particular, the null coupled (type IV) configurations. This is shown to be consistent with the equations of motion, insuring that the two metrics admit compatible notions of space and time.

\subsection{Choice of square root matrix from general covariance}

The bimetric equations of motion have the form, ${ }^{10}$

$$
\left(G^{g}\right)^{\mu}{ }_{\nu}+\sum_{n=0}^{3} c_{n}^{g}\left(S^{n}\right)^{\mu}{ }_{\nu}=0, \quad\left(G^{f}\right)^{\mu}{ }_{\nu}+\sum_{n=0}^{3} c_{n}^{f}\left(S^{n}\right)^{\mu}{ }_{\nu}=0
$$

where $G^{g}$ and $G^{f}$ denote the Einstein tensors for $g$ and $f$ metrics, respectively, and the coefficients $c_{n}^{g}$ and $c_{n}^{f}$ are functions of $\operatorname{Tr}\left(S^{m}\right)$. Under general coordinate transformations $x^{\mu} \rightarrow \tilde{x}^{\mu}$, the matrix $A^{\mu}{ }_{\nu}=g^{\mu \lambda} f_{\lambda \nu}$ transforms as a $(1,1)$ tensor to $\tilde{A}(\tilde{x})=Q^{-1} A(x) Q$, where $Q^{\mu}{ }_{\nu}=\partial x^{\mu} / \partial \tilde{x}^{\nu}$. The bimetric equations will transform covariantly only if $S=\sqrt{A}$ also transforms as a $(1,1)$ tensor, which requires that,

$$
\sqrt{Q^{-1} A Q}=Q^{-1} \sqrt{A} Q
$$

Nonprimary square roots do not satisfy this property and hence can be discarded by the requirement of general covariance. We now show that this leads to a unique choice for the square root matrix.

The nature of the square root of $A$ is determined by its Jordan normal form $A_{\mathrm{J}}$ (see appendix A). First, note that for Type IV configurations, $A_{\mathrm{J}}=\operatorname{diag}\left(-\lambda,-\lambda, \lambda_{3}, \lambda_{4}\right)$ and its primary square roots contain the $2 \times 2$ blocks $\pm \operatorname{diag}\left(i \lambda^{1 / 2}, i \lambda^{1 / 2}\right)$. These are nonreal in a real basis and are ruled out by the reality condition. On the other hand, square roots containing the $2 \times 2$ blocks $\pm \operatorname{diag}\left(i \lambda^{1 / 2},-i \lambda^{1 / 2}\right)$ have a real form in an appropriate basis which is the reason why Type IV metrics are not ruled out by the reality condition. However, the latter roots are nonprimary and violate general covariance. Thus, Type IV configurations do not admit real square roots that preserve general covariance.

In the remaining Type I-III configurations, $A$ has no negative real eigenvalue, hence it admits multiple real primary square roots. Let us denote its distinct eigenvalues by $\lambda_{i}$ and the corresponding Jordan blocks by $J_{i}$ (there are 2 to 4 such blocks depending on the configuration). Then, $A_{\mathrm{J}}=\operatorname{diag}\left(J_{1}, \cdots, J_{s}\right)$. Assuming that the eigenvalues in different Jordan blocks are distinct, the different primary square roots are,

$$
\sqrt{A_{\mathrm{J}}}=\operatorname{diag}\left( \pm J_{1}^{1 / 2}, \cdots, \pm J_{s}^{1 / 2}\right) .
$$

Here, $J_{i}^{1 / 2}$ are given by eq. (A.3) and the \pm signs are chosen independently. Selecting the + sign for all blocks gives the principal square root. (For the purpose of this argument we do not distinguish between the principal roots $\sqrt{A}$ and $-\sqrt{A}$ as the extra overall sign can be absorbed in the $\beta_{n}$ parameters of the theory.) Other combinations of the signs produce the remaining primary square roots as long as the $\lambda_{i}$ remain distinct. However, it is possible that due to symmetries, or during the evolution, two eigenvalues collapse to the

\footnotetext{
${ }^{10}$ For an explicit derivation of the equations, see, for example, the appendix in [45].
} 

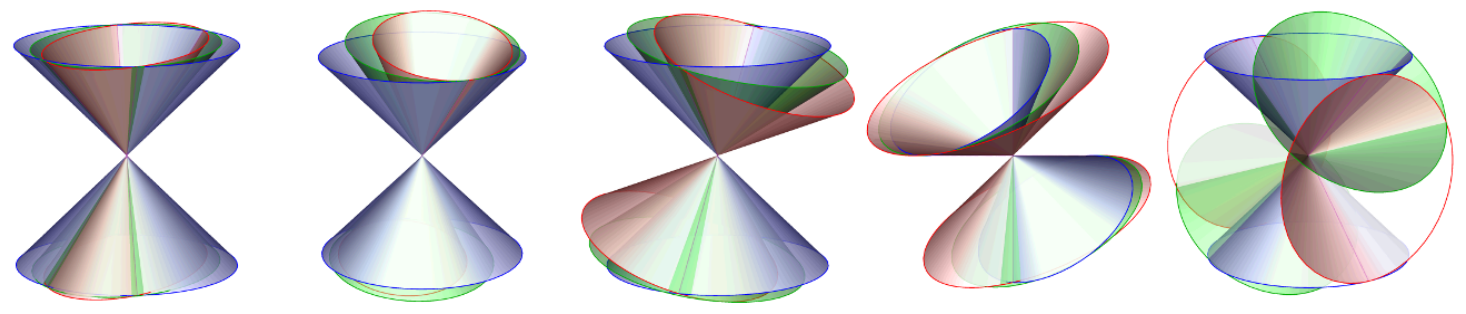

Figure 5. The null cones of $h$, in green, relative to $f$ and $g$.

same value, say, $\lambda_{k}=\lambda_{l}$, without producing a discontinuity in the square root. Then, if the corresponding $J_{k}^{1 / 2}$ and $J_{l}^{1 / 2}$ in eq. (3.3) have opposite signs, the square root becomes nonprimary. Thus, nonprincipal square roots become nonprimary whenever eigenvalues in blocks with different signs in (3.3) become equal. Only the principal square root avoids becoming nonprimary for the allowed range of $\lambda_{i}$, since all blocks $J_{i}^{1 / 2}$ are guaranteed to have the same sign.

To summarize, requiring reality and general covariance of the equations restricts $\sqrt{g^{-1} f}$ uniquely to be the principal real square root which is always a primary matrix function. In particular, Type IV configurations are disallowed since their only real square roots are nonprimary. The allowed configurations are the causally coupled metrics (Type I-III) which always admit compatible $3+1$ decompositions. In subsection 3.3 we will show that disallowing Type IV metrics is consistent with the equations of motion and is, in fact, also required by the variational principle.

\subsection{Geometric significance of the principal root}

To see the geometrical meaning of the principal square root, consider the covariant tensor,

$$
h_{\mu \nu}=g_{\mu \rho}\left(\sqrt{g^{-1} f}\right)_{\nu}^{\rho},
$$

which is symmetric and nonsingular. For the principal square root, $h$ is Lorentzian with the same signature as $g$ and $f$, and its null cone contains the intersection of the null cones of $g$ and $f$. This is depicted in figure 5, for Type I-IV metrics, with the $h$ null cone shown in green. The explicit expressions are given in appendix B. The metric $h$ can be regarded as the geometric mean of $g$ and $f$, as shown in appendix D (for a more detailed analysis of $h_{\mu \nu}$ see [21]). ${ }^{11}$ As discussed above, $h$ is not a tensor in Type IV.

This property of $h_{\mu \nu}$ can be exploited to find coordinates compatible with the $3+1$ decompositions of both metrics. In general relativity, as null cones tilt during evolution, one may need to adjust the coordinate system to maintain a proper $3+1$ decomposition, for example, across a blackhole horizon. In bimetric theory similar situations arise also for the relative tilt of the two null cones, as illustrated in figure 6 . Let us start with a coordinate system where both metrics admit proper $3+1$ decompositions in figure 6 (a), i.e., $g^{00}<0$ and $f^{00}<0$. In Gaussian coordinates adapted to $g$, a 'horizontal' hypersurface (the dashed

\footnotetext{
${ }^{11}$ At the linearized level, $h$ is a combination of massive and massless modes [22]. But minimal coupling of matter to $h$ is not ghost-free since the vacuum energy contribution $\sqrt{|\operatorname{det} h|}=(|\operatorname{det} g / \operatorname{det} f|)^{1 / 4}$ is not found in the ghost-free bimetric potential.
} 


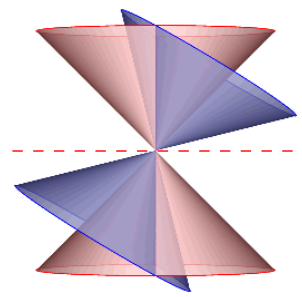

(a)

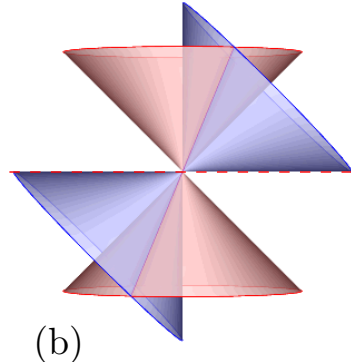

(b)

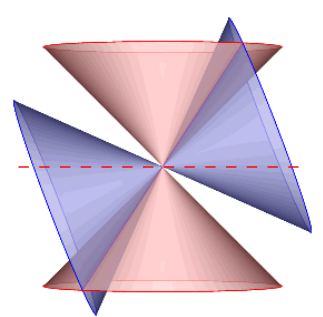

(c)

Figure 6. Good and bad coordinate systems.

line) is initially spacelike with respect to both metrics. As the $f$ null cone tilts and hits the spacelike surface (b), its lapse and shifts become infinite simultaneously. Beyond that, in (c), its lapse becomes imaginary $\left(f^{00}>0\right)$, and $f_{i j}$ becomes indefinite. This can be avoided by adapting the coordinates to $h$ instead. Namely, a time direction at the 'center' of the $h$ null cone is always within the intersection of the $g$ and $f$ null cones, and a hypersurface transverse to it is always spacelike with respect to all three metrics. One can implement this by starting from the local coordinates of subsection 2.3 .2 which puts $f$ and $g$ in the canonical pair form, and then change to a new basis where $h$ is, say, in Gaussian normal form. In such a basis, both $f$ and $g$ will have a proper $3+1$ decomposition. ${ }^{12}$ Implementing this in massive gravity with a fixed $f_{\mu \nu}$ requires the Stückelberg fields.

\subsection{Type IV metrics as limits of Type IIb}

Type IV configurations are not an isolated class in the algebraic sense, but arise as limits of Type IIb metrics. Here we point out that when Type IIb metrics degenerate into Type IV, $S=\sqrt{g^{-1} f}$ develops a branch cut. As will be argued in the next subsection, a consequence is that Type IV metrics are not valid solutions in bimetric theory.

Type IIb metrics arise when $g^{-1} f$ has complex eigenvalues, $a \pm \mathrm{i} b=\lambda(\cos \theta \pm \mathrm{i} \sin \theta)$, with $b \neq 0$ or $\lambda>0, \theta \neq 0, \pi$. Then, in a real Jordan basis, equation (2.12) gives,

$$
g^{-1} f=\operatorname{diag}\left(\left(\begin{array}{rr}
a & -b \\
b & a
\end{array}\right), \lambda_{2}, \lambda_{3}\right)=\operatorname{diag}\left(\lambda\left(\begin{array}{rr}
\cos \theta & -\sin \theta \\
\sin \theta & \cos \theta
\end{array}\right), \lambda_{2}, \lambda_{3}\right) .
$$

For brevity, we again use $g$ for $Z^{\top} g Z$, etc., since the $Z$ factors do not alter the outcome. The nontrivial block of this is a rotation matrix. On the other hand, Type IV metrics (2.10) arise when $g^{-1} f$ has a pair of real negative eigenvalues,

$$
g^{-1} f=\operatorname{diag}\left(-\lambda,-\lambda, \lambda_{2}, \lambda_{3}\right), \quad \text { with } \quad \lambda, \lambda_{2}, \lambda_{3}>0 .
$$

\footnotetext{
${ }^{12}$ It is instructive to revisit the ghost analysis of [15] in the light of this discussion. There, initially one starts with a $3+1$ type decomposition without specifying the sign of $f^{00}$. The decomposition is proper only if $f^{00}<0$ so that the lapse $M=\left(-f^{00}\right)^{-1 / 2}$ is real. Then a real square root exists if $x=-f^{00}-n^{i} f_{i j} n^{j}>0$ for the given $n^{i}$ (see eqs. (3.22) and (3.30) in [15], also [46]). However, $x>0$ holds not only for proper $3+1$ decompositions but also when $f^{00}>0$, since then $f_{i j}$ is indefinite. The present analysis shows that this case corresponds to a bad coordinate choice, and that at long as $x>0$, a frame always exists in which the decomposition is proper.
} 


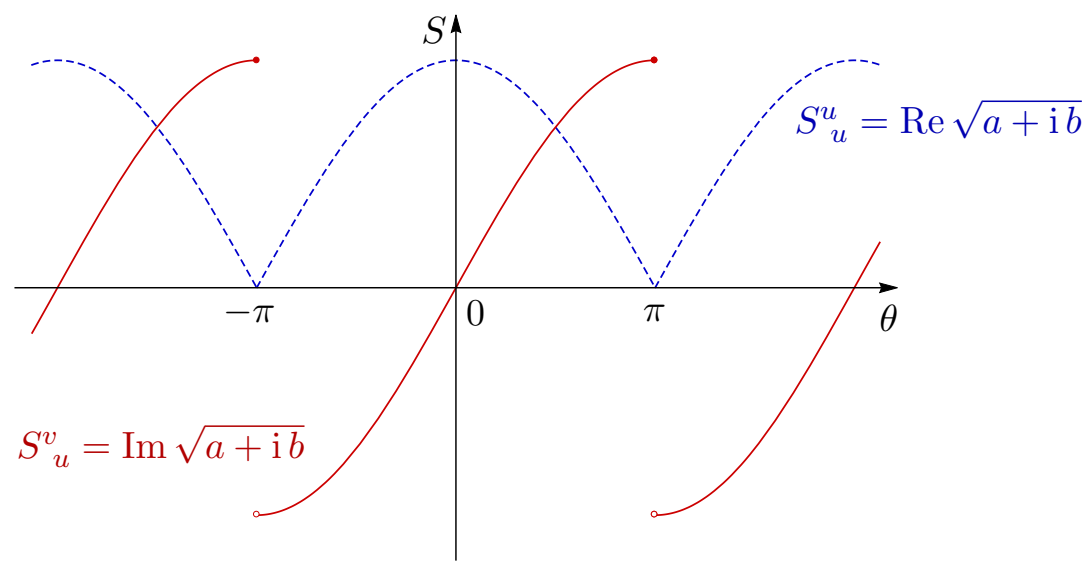

Figure 7. Type IV $(\theta=\pi)$ as a branch cut of Type IIb.

Clearly, Type IV can arise from Type IIb when $\theta \rightarrow \pi$, or equivalently, $a<0$ and $b \rightarrow 0$ (the limit $\theta \rightarrow 0$ leading to Type I is harmless here).

We are interested in $S=\sqrt{g^{-1} f}$ in this limit. Let us first consider the square root $\sqrt{a+\mathrm{i} b} \equiv \sqrt{\lambda} \sqrt{\cos \theta+\mathrm{i} \sin \theta}$. On the principal branch it has the real and imaginary parts, ${ }^{13}$

$$
\begin{aligned}
& \operatorname{Re} \sqrt{a+\mathrm{i} b}=\frac{1}{\sqrt{2}} \sqrt{\sqrt{a^{2}+b^{2}}+a}=\sqrt{\lambda} \operatorname{sgn}\left(\cos \frac{\theta}{2}\right) \cos \frac{\theta}{2}, \\
& \operatorname{Im} \sqrt{a+\mathrm{i} b}=\frac{1}{\sqrt{2}} \operatorname{sgn}(b) \sqrt{\sqrt{a^{2}+b^{2}}-a}=\sqrt{\lambda} \operatorname{sgn}\left(\cos \frac{\theta}{2}\right) \sin \frac{\theta}{2} .
\end{aligned}
$$

Here, $\sqrt{z}$ denotes the principal root of $z$ (another branch of the square root is $-\sqrt{a+\mathrm{i} b}$ ). These quantities are plotted in figure 7 , for $0 \leq \theta \leq 2 \pi$. The quantity $\operatorname{Im} \sqrt{a+\mathrm{i} b}$ is discontinuous at $\theta=\pi$, corresponding to the branch cut along the real negative axis on the complex plane, whereas $\operatorname{Re} \sqrt{a+\mathrm{i} b}$ is continuous but not differentiable at the branch cut. By convention, the square root of -1 is chosen as $+i$.

Now, the principal square root of $g^{-1} f$ for Type IIb configurations (3.5) can be explicitly evaluated (see also [33, 35]), and contains the $2 \times 2$ block,

$$
\left(\begin{array}{cc}
S_{u}^{u} & S_{v}^{u} \\
S_{u}^{v} & S_{v}^{v}
\end{array}\right)=\sqrt{\left(\begin{array}{rr}
a & -b \\
b & a
\end{array}\right)}=\left(\begin{array}{rr}
\operatorname{Re} \sqrt{a+\mathrm{i} b} & -\operatorname{Im} \sqrt{a+\mathrm{i} b} \\
\operatorname{Im} \sqrt{a+\mathrm{i} b} & \operatorname{Re} \sqrt{a+\mathrm{i} b}
\end{array}\right)
$$

Here, $u$ and $v$ are the null cone coordinates $x^{0} \pm x^{1}$ corresponding to the form of $g_{\mu \nu}$ in (2.12). From figure 7 it is clear that at $\theta=\pi$, when Type IIb goes over to Type IV, the diagonal elements $S_{u}^{u}, S_{v}{ }_{v}$ are not smooth while the off-diagonal elements $S_{v}^{u}, S_{u}{ }_{u}$ are discontinuous. Thus, at $\theta=\pi$ (Type IV), the variation of the square root matrix $S$ with respect to $\theta$ is not defined. The same holds for the bimetric potential $V(S)$.

\footnotetext{
${ }^{13}$ Recall that on the principal branch, the real part of $\sqrt{a+\mathrm{i} b}$ is positive while its imaginary part has the same sign as $b$. Thus, the principal square root of $\mathrm{e}^{\mathrm{i} \theta}$ is $\operatorname{sgn}\left(\cos \frac{\theta}{2}\right) \mathrm{e}^{\mathrm{i} \theta / 2}$ and not just $\mathrm{e}^{\mathrm{i} \theta / 2}$.
} 


\subsection{The absence of null coupled (Type IV) configurations}

So far we have argued that Type IV configurations could be discarded on the ground that they lead to the violation of general covariance. This justifies not using them as initial conditions, or as ansatz for finding solutions. However, since Type IV metrics can also arise as limits of Type IIb, they can be discarded consistently only if the equations of motion do not evolve Type IIb metrics into Type IV. Here we show that this is indeed the case and that the theory does not admit null coupled (Type IV) configurations as solutions due to the square root branch cut. This is illustrated with a simple example from mechanics.

The variation of the bimetric action (1.7) with respect to continuous functions $g_{\mu \nu}$ has the form $\delta_{g} \mathcal{S}=\int d^{4} x\left(G_{\mu \nu}^{g}+V_{\mu \nu}^{g}\right) \delta g^{\mu \nu}$, where $G_{\mu \nu}^{g}$ is the Einstein tensor and $V_{\mu \nu}^{g}$ arises from the variation of the potential $\sqrt{-g} V$. Provided that $V_{\mu \nu}^{g}$ are also continuous functions, the fundamental lemma of calculus of variations states that requiring $\delta_{g} \mathcal{S}=0$ leads to the equations of motion (similarly for $f_{\mu \nu}$ ),

$$
G_{\mu \nu}^{g}+V_{\mu \nu}^{g}=0, \quad G_{\mu \nu}^{f}+V_{\mu \nu}^{f}=0 .
$$

Let us assume that these admit solutions $g$ and $f$ that evolve into Type IV configurations in some region $\Sigma$ of spacetime. But in this region, the potential $V$ could not have been varied to produce the $V_{\mu \nu}^{g}\left(V_{\mu \nu}^{f}\right)$ in $\delta_{g} \mathcal{S}\left(\delta_{f} \mathcal{S}\right)$, hence the above equations do not apply in $\Sigma$. This is a consequence of $S=\sqrt{g^{-1} f}$ being the principal square root where Type IV coincides with a branch cut.

In the parameterization of the previous subsection, the branch cut is at $\theta(x)=\pi$ which, generically, corresponds to a 3 dimensional hypersurface $\Sigma$ characterized by the normal $\partial_{\mu} \theta(x)$. Since the variation of $\sqrt{-g} V(S)$ is discontinuous at $\theta(x)=\pi$, the variational principle can be applied piecewise for $\theta(x)<\pi$ and $\theta(x)>\pi$, or formally, on both sides of the hypersurface defined by $\theta(x)=\pi$, to yield two sets of equations of motion,

$$
\begin{array}{lll}
\theta<\pi: & G_{\mu \nu}^{g}+V_{\mu \nu}^{g}\left(S_{<}\right)=0, & G_{\mu \nu}^{f}+V_{\mu \nu}^{f}\left(S_{<}\right)=0, \\
\theta>\pi: & G_{\mu \nu}^{g}+V_{\mu \nu}^{g}\left(S_{>}\right)=0, & G_{\mu \nu}^{f}+V_{\mu \nu}^{f}\left(S_{>}\right)=0,
\end{array}
$$

where $S_{<}$and $S_{>}$are $S$ evaluated on two sides of the branch cut. But there are no equation valid at $\theta=\pi$. Note that the Bianchi constraints $\nabla_{\mu}^{g} V^{g \mu}{ }_{\nu}=0$ and $\nabla_{\mu}^{f} V^{f \mu}{ }_{\nu}=0$ are also not valid at $\theta=\pi$. The variational principle does not lead to equations of motion valid for Type IV metrics showing that such configurations are not valid solutions of bimetric equations.

If a Type IIb solution, say, for $\theta<\pi$, approaches Type IV, it must either be reflected back at the branch cut, or must be matched across $\theta=\pi$ to another Type IIb configuration for $\theta>\pi$, in the spirit of junction conditions with no need for equations valid at $\theta=\pi$. Which of the two scenarios is actually realized is not addressed here. Matching Type IIb configurations across Type IV could potentially lead to a problem with the relative time orientation of the two metrics, but restricting $S$ to a single branch also preserves the relative time orientations (as discussed in appendix E).

Here we do not attempt to analyze the behavior of bimetric solutions near the branch cut in more detail, but it is instructive to illustrate the situation with an example. A simple 
mechanical system displaying a similar behavior is given by the action,

$$
A=\int \mathrm{d} t\left(\dot{x}^{2} / 2-\lambda \sqrt{x^{2}}\right)
$$

defined on the principal branch of $\sqrt{x^{2}}=|x|$. This describes a charge in a constant electric field along the $x$-axis which abruptly reverses direction at $x=0$. The variation of the action is discontinuous at $x=0$ and the variational principle yields the equation of motion,

$$
\ddot{x}=-\lambda \quad(x>0), \quad \ddot{x}=\lambda \quad(x<0),
$$

with no equation valid at $x=0$. However, continuous solutions can be easily obtained by matching the two parabolic solutions at $x=0$. For $\lambda<0$, the solution crosses $x=0$ at most once. For $\lambda>0$, the solution $x(t)$ is an array of alternating concave and convex parabolas matched at $x=0$, describing a particle oscillating about $x=0$.

In the mechanical system (3.14), the discontinuity at $x=0$ is to be regarded as the outcome of a coarse graining approximation. At the microscopic level, the electric field of strength $\lambda$ cannot be reversed abruptly but will change direction over a thin layer around $x=0$. Equations (3.14) then provide an effective description at large distances. In analogy, the appearance of Type IV discontinuity may be regarded as an indication of the effective nature of bimetric theory, signaling the need for new degrees of freedom at a more fundamental level. ${ }^{14}$

Finally, it is worth noting that in the analysis of perturbations in massive gravity [47], one needs to solve the matrix equation $\delta\left(S^{2}\right)=S \delta S+\delta S S$ to express $\delta S$ in terms of $\delta\left(S^{2}\right)$. By a theorem of Sylvester, a unique solution exists if and only if $\sigma(S) \cap \sigma(-S)=\varnothing$, i.e., when $S$ and $-S$ have no common eigenvalues. This property is not satisfied by Type IV metrics, which were then excluded from the analysis. Another implication of Sylvester's theorem is that for general perturbations $\delta g_{\mu \nu}$ and $\delta f_{\mu \nu}$ around Type I-III configurations, a corresponding $\delta S$ exists, hence, these configurations are not isolated.

\section{Remarks on causality and superluminality}

To put our results in perspective, we briefly comment on the problems of causality and superluminality in bimetric theory. We also discuss the implications for some studies of acausality in massive gravity [48-52]. For convenience, the condition for bimetric theory to have meaningful dynamics can be stated in two steps.

- The covariant equations (3.10) must be writable as time evolution equations with spatial hypersurfaces for specifying initial data, at least locally. As discussed earlier, this is not self-evident in a bimetric setup.

- The initial data must uniquely determine the solutions at later times, thereby implying causality.

\footnotetext{
${ }^{14}$ We would like to thank K. Izumi and Y. C. Ong for a discussion of this issue.
} 
We have addressed the first requirement, showing that the theory admits $3+1$ decompositions compatible with both metrics. This provides the necessary setup for addressing the second condition, but the well-posedness of the initial value problem is not addressed here and remains an open question. However, one can already note aspects of the bimetric theory that differ from general relativity, but may not necessarily imply lack of causality.

If the initial conditions (specified over an appropriate domain of dependence) uniquely determine the solution at later times then the dynamics will be causal even if the theory contains "faster than light" propagation, as explained in detail in [53]. In such cases, the equations will determine a causal cone which need not coincide with the null cone of a gravitational metric, say, $g_{\mu \nu}$. Then, superluminality with respect to $g_{\mu \nu}$ will not necessarily indicate causality violation. In traditional single metric theories, including in general relativity, the equations of motion encode a causal cone that coincides with the null cone of $g_{\mu \nu}$ which thus determines the causal structure. However, as emphasized in [53], this need not be the case in more general setups. In [54] the possibility of superluminal propagation without causality violation has been discussed in a class of scalar field theories. For discussions of similar issues in some other bimetric scenarios see $[55,56]$.

In the ghost-free bimetric theory, one expects that the Einstein-Hilbert term for each metric allows for propagation at least within the null cone of that metric. This alone means that, whenever the two null cones do not coincide, the theory could allow for the propagation of, say, $f$-metric perturbations or matter, outside the null cone of the $g$-metric, and vice versa. Interactions introduce further complexities, but spacelike propagation with respect to either or both metrics is still expected. However, as indicated above, this is not necessarily a violation of causality, provided the initial value problem is well-posed. This problem has not yet been analyzed in a conclusive way for bimetric theory. For most practical purposes, where the theory should be close to GR, the effects of propagation outside the null cone of the gravitational metric can be suppressed. Also, since observable matter couples minimally only to a single metric, say, $g_{\mu \nu}$, in a ghost-free way, it is primarily sensitive to the null cone of this gravitational metric. For cosmologically relevant solutions, the $g$ and $f$ null cones approach each other at late times.

In any case, it should be emphasized that the bimetric potential is the spin-2 analogue of the Proca mass for vector fields, $\sqrt{-g} g^{\mu \nu} A_{\mu} A_{\nu}$, indicating that the theory may be incomplete in the absence of some extra Higgs-like degrees of freedom. It is possible that, in the spin-2 case, ignoring the extra degrees of freedom could cause pathologies even at the classical level. One may also have to further restrict the allowed solutions by imposing the analog of energy and hyperbolicity conditions, as in general relativity.

\subsection{On the studies of acausality in massive gravity}

Here, after a brief review of massive gravity in the vielbein formulation and of its initial value formulation, we point out that a number of arguments for acausal propagation in the theory is based on the Type IV and other inadmissible configurations. Thus more work, perhaps on the lines of [48], is needed to clarify the causal properties of the theory (see also $[57,58])$. 
Massive gravity theories can arise as limits of bimetric theory $\left(m_{f} \rightarrow \infty\right.$ at fixed $\left.m_{g}\right)$ around classes of solutions that admit such a limit [59]. In practice, one simply drops $\sqrt{|f|} R_{f}$ from the bimetric action (1.7) and treats $f=\bar{f}$ as nondynamical (e.g., $\bar{f}=\eta$, [6] or any fixed $\bar{f},[14,15]) .{ }^{15}$ In terms of vielbeins $e_{\mu}^{a}$ and $d_{\mu}^{a}$, corresponding to metrics $g_{\mu \nu}$ and $f_{\mu \nu}$, the interaction potential becomes [4, 60],

$$
\sum_{n=0}^{4} \beta_{n} \epsilon_{a_{1} \cdots a_{n} a_{n+1} \cdots a_{4}} \epsilon^{\mu_{1} \cdots \mu_{n} \mu_{n+1} \cdots \mu_{4}} d_{\mu_{1}}^{a_{1}} \cdots d_{\mu_{n}}^{a_{n}} e_{\mu_{n+1}}^{a_{n+1}} \cdots e_{\mu_{4}}^{a_{4}}
$$

A symmetrization constraint, $d_{\mu[a} e_{b]}^{\mu}=0$, implies $\sqrt{g^{-1} f}=e^{-1} d$, insuring equivalence to the metric formulation. Although the vielbein form seems simpler to manipulate, in reality, solving the symmetrization constraint involves the same level of complication as computing a square root matrix. When $f$ is nondynamical, there is no a priori requirement for using compatible $3+1$ decompositions for $f$ and $g$. However, such decompositions always exist and must be employed when the theory is regarded as a bimetric limit. Below we comment on some analysis of the initial value problem, superluminality, and acausality in massive gravity, carried out mostly in the vielbein formulation.

The initial value problem in massive gravity was addressed in [48], by selecting a time direction and expressing the equations in the quasilinear form $A_{a b} \partial_{t} \phi^{b}=F_{a}$, where $A_{a b}$ and $F_{a}$ are functions of the dynamical fields $\phi^{a}$ and their spatial derivatives. The well-posedness of the initial value problem then requires that the matrix $A$ is invertible, so that all $\phi^{a}$ have well-defined evolution equations. However, if the theory admits field configurations such that $\operatorname{det} A=0$, then time evolution will not be well-defined. Further analysis can shed light on whether valid acausal configurations can arise in massive gravity, or if further restrictions are needed. In any case, the analysis of [48] shows that configurations with $\operatorname{det} A=0$ are not a generic feature of the theory, as had been suggested earlier.

Superluminality and acausality in massive gravity have been studied in reference [49] and related works by searching for characteristic surfaces (corresponding to maximum speed signals) that are spacelike with respect to $g_{\mu \nu}$. Propagation along such a surface would be superluminal with respect to $g_{\mu \nu}$. Although this, by itself, does not imply acausality, [49] argues that specific examples can be constructed where superluminality leads to the formation of local Closed Causal Curves (CCC). Here, we show that these arguments rely on disallowed field configurations corresponding to the nonprincipal square roots.

Spacelike characteristic surfaces (with timelike normals) exist if an associated characteristic matrix, say, $\Delta$, obtained from the equations of motion, satisfies $\operatorname{det} \Delta=0 .{ }^{16}$ Reference [49] contains two examples of field configurations that are supposed to give $\operatorname{det} \Delta=0$. In the first example, this condition reduces to $-3 m^{2} / 2+l_{00} K^{j} K_{j}=0$, for some vectors $K^{j}$. It is stated that if the normal to the characteristic surface (which has

\footnotetext{
${ }^{15}$ If massive gravity is to have $g \propto \bar{f}$ as a solution, then $\bar{f}$ must solve Einstein's equation and only then, perturbations $\delta g_{\mu \nu}$ will have a Fierz-Pauli mass. An Einstein equation for $\bar{f}$ arises in the massive gravity limit of bimetric equations, but not from the massive gravity action.

${ }^{16}$ In general, the well-posedness of the initial value problem requires the vanishing of the characteristic determinant. This also determines the causal cone for the problem, see, for example, [61]. The analysis of characteristics in [49] has a more limited scope.
} 
been taken to be timelike with respect to $\left.g_{\mu \nu}\right)$ is not timelike with respect to $f_{\mu \nu}$, then $l_{00}$ is positive. Then the equation becomes a difference of positive terms and can be satisfied; hence the superluminality. However, this scenario is not realized by the allowed configurations. In our conventions, $l_{\mu \nu}=\left(g h^{-1} g\right)_{\mu \nu}$, where $h=g S$ is the metric in (3.4), depicted in figure 5. As is obvious from the figures (and can be explicitly verified from the equations in full generality), any coordinate system in which $g$ and $f$ admit compatible $3+1$ decompositions leads to $l_{00}<0$. For example, in Gaussian normal coordinates adapted to $g, l_{00}=h^{00}=-N_{h}^{-2}<0$, where $N_{h}$ denotes the lapse of $h$. Obviously, $l_{00}=h^{00}<0$ as long as the spatial hypersurface is spacelike with respect to $h_{\mu \nu}$ (even for a time direction outside the null cone of $h$ ). As the $h$ null cone tilts away from the $g$ null cone, the $f$ null cone will hit the spatial hypersurface (invalidating the coordinate system choice) before $l_{00}$ turns positive. Hence, in this example, the superluminality constraint is not satisfied.

The second example in [49] is about the formation of Closed Causal Curves (CCC), which would imply violation of local causality. This is elaborated further in [50]. However, this construction requires that $g$ and $f$ are Type IV metrics which, as shown above in section 3 , are not valid solutions of the theory.

Reference [52] provides new examples of backgrounds that exhibit superluminality and Closed Causal Curves, and which are not Type IV configurations. However, these correspond to choosing a nonprincipal square root which is ruled out if massive gravity is regarded as a limit of bimetric theory (but could be allowed if no extra symmetries are required). These examples amount to choosing the vielbeins as $\left(e_{\mu}^{a}\right)=\operatorname{diag}(A, B, C,-B)$ and $\left(d^{a}{ }_{\mu}\right)=\operatorname{diag}(1,1,1,1)$. Note that the metric $g=e^{\top} \eta e$ does not distinguish between vielbein $e$ above and some $e^{\prime}=\operatorname{diag}(A, B, C, B)$ which result in opposite volume orientations $\operatorname{det}(e)=-\operatorname{det}\left(e^{\prime}\right)$. Also in both cases, $S^{2}=g^{-1} f=\operatorname{diag}\left(A^{-2}, B^{-2}, C^{-2}, B^{-2}\right)$. But the square root matrix $S$ is sensitive to the difference; $S=e^{\prime-1} d$ is a primary square root, whereas $S=e^{-1} d$ is a nonprimary one which we have ruled out. ${ }^{17}$

Hence, to conclude this section, the above references do not prove that massive gravity violates causality through the formation of local CCCs, especially, when the theory is regarded as a limit of bimetric theory and defined as outlined above. Settling the question of causality in bimetric theory requires more accurate analysis that takes into account the validity of the field configurations, and of the $3+1$ decompositions. For alternative approaches see, for example, [62].

\section{Discussion}

Here we briefly highlight some ramifications and limitations of our results, and the relation to the vielbein formulations.

The above results show the validity of the proofs of the absence of the BD ghosts in massive gravity and bimetric theory. The proofs were carried out in the Hamiltonian formulation assuming that the two metrics admitted simultaneous $3+1$ decompositions [79, 15, 63] (for a detailed analysis see [64]). The analysis here shows that this is fully

\footnotetext{
${ }^{17}$ The statement in [52], that a bimetric theory cannot be consistently coupled to matter without destroying its degree of freedom count, is inaccurate since ghost-free matter couplings are well known [8].
} 
adequate since all allowed configurations admit such a decomposition. The ghost analysis has also been carried out in covariant forms for massive gravity [19, 51] and, bimetric theory [65]. In these analyses too, except for massive gravity models with $\beta_{3}=0$, one needs to invoke $3+1$ decomposition to verify the validity of the constraints.

Early studies of ghost in massive gravity involved working with a binomial series expansion of $\sqrt{\mathbb{1}+H}$ that holds for configurations perturbatively close to $g_{\mu \nu}=\eta_{\mu \nu}$ [6]. As long as $H$ remains perturbative, this is consistent with restricting the square root to the principal branch (since $1-x$ and $|1-x|$ are the same for $x<1$ but not for $x>1$ ). However, if the square root is defined without restricting to a specific branch (so that $\sqrt{\mathrm{e}^{\mathrm{i} \theta}}=\mathrm{e}^{\mathrm{i} \theta / 2}$, rather than $\sqrt{\mathrm{e}^{\mathrm{i} \theta}}=\left|\mathrm{e}^{\mathrm{i} \theta / 2}\right|$ ), then its variation is continuous and Type IV metrics become regular solutions of the field equations. Restricting the square root to the principal branch avoids this problem. The choice of the branch for the square root matrix may also be restricted by ghost considerations and by requiring backgrounds with certain symmetries as in [70] for the case of Lorentz invariant massive gravity.

The results also clarify the meaning of the vielbein symmetrization condition $d_{\mu[a} e_{b]}^{\mu}=$ 0 [4], sometimes called the Deser-van Nieuwenhuizen gauge, although it is known not to be a gauge condition. Since solving this condition is equivalent to finding the matrix square root $S$ [66], it involves the same challenges of multiple solutions and the choice of branch, with the same resolutions as proposed here. Our results show that the symmetrization condition can be regarded as a gauge condition only when the two vielbeins have intersecting nullcones. Real solutions do not exist otherwise. This also provides a physical justification for imposing the symmetrization condition by hand in theories where they are not necessarily implied by the equations of motion, such as in the vielbein formulation of bimetric theory with all $\beta_{n} \neq 0,[60,67]$ or in [68]. Conversely, when a vielbein pair cannot be symmetrized by a Lorentz transformation, the corresponding null-cones do not intersect in the ways described here.

The presented Theorem 2.3, which connects the algebraic existence of the square root $\sqrt{g^{-1} f}$ with the local geometrical relations between the null cones of $f$ and $g$, is actually more general. In fact, the proof applies to any function $F\left(g^{-1} f\right)$ which requires that the eigenvalues of $g^{-1} f$ are not on $\mathbb{R}^{-}$. For example, $F(X)$ can be taken as the function $X^{\xi} \equiv \exp (\xi \log X)$ for a real $\xi$.

As emphasized, the statements in this paper are local and can serve as a first step towards studying causality and the initial value problem at the global level. Since global hyperbolicity is a reasonable condition in the context of GR, the logical extension would be to define a notion of simultaneous global hyperbolicity for a manifold equipped with two metrics and consistent with the bimetric equations of motion. Causality in massive gravity has recently been considered in [62] from a different point of view.

Also, as pointed out, the appearance of the branch cut associated with Type IV (and the analogy with a discontinuous electric field) could be an indication of the effective nature of the theory and could shed light on the nature of the extra degrees of freedom that have been integrated out. Then one expects that this may also have ramifications for the specific form of the quantum completion of the theory [69]. 


\section{Acknowledgments}

We would like to thank Keisuke Izumi, Yen Chin Ong, Angnis Schmidt-May, Kjell Rosquist, Lars Andersson, Anders Lundkvist, and Luis Apolo for helpful discussions. We are grateful to Mikael von Strauss for a careful reading of the manuscript. We would also like to thank the organizers of the PI conference on "Superluminality in Effective Field Theories for Cosmology" 2015, where the results in section 2 were first presented. Subsequently, the results have been presented in other workshops and conferneces, including FANCY 2016 in Odense, Indian Strings Meeting 2016 in Pune, and a number of Nordita workshops.

\section{A The square root matrix function}

Here we collect some properties of the square root matrix function that are used in the text of the paper (see, for example, [33]). Given an ordinary scalar function $F(x)$ and a matrix $A$, one can construct a matrix function $F(A)$. An example is $F(x)=\sqrt{x}$, leading to the matrix square root, $F(A)=\sqrt{A}$. To make this precise, consider transforming $A$ to its Jordan normal form, $Z^{-1} A Z=\operatorname{diag}\left(J_{1}, \ldots, \ldots, J_{s}\right)$, where $J_{i} \equiv J_{i}\left(\lambda_{i}\right)$ is the Jordan block corresponding to the eigenvalue $\lambda_{i}$ which could be real or complex. Then, the precise meaning of $F(A)$ is a matrix which, in the same Jordan basis, reads (Definitions 1.2 in [33] and 6.2.4 in [35]),

$$
Z^{-1} F(A) Z=\operatorname{diag}\left(F\left(J_{1}\right), \ldots, \ldots, F\left(J_{p}\right)\right)
$$

where,

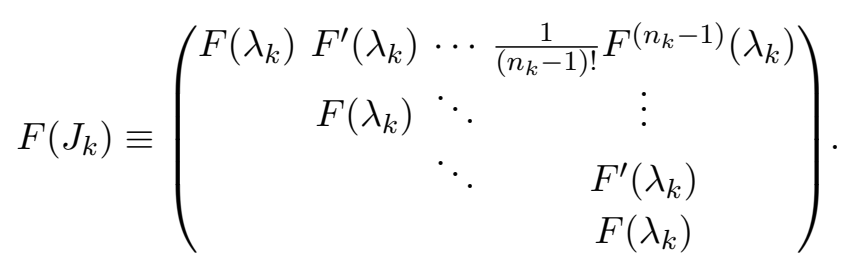

Here $F\left(\lambda_{k}\right)$ is the scalar function and $F^{(n)}$ denotes its $n$-th derivative. When the stem function $F(x)$ has multiple branches, the same branch must be chosen within each block.

For $F(x)=x^{1 / 2}$, equation (A.1) defines the matrix function $F(A)=\sqrt{A}$, with,

$$
s F\left(J_{k}\right)= \pm\left(\begin{array}{cccc}
\lambda_{k}^{1 / 2} & \frac{1}{2} \lambda_{k}^{-1 / 2} & \cdots & (-1)^{n_{k}} \frac{\left(2 n_{k}-5\right) ! !}{2^{n_{k}-1}} \lambda_{k}^{3 / 2-n_{k}} \\
& \lambda_{k}^{1 / 2} & \ddots & \vdots \\
& & \ddots & \frac{1}{2} \lambda_{k}^{-1 / 2} \\
& & & \lambda_{k}^{1 / 2}
\end{array}\right)
$$

The \pm signs in front of the matrix reflect explicitly the possible choices of branch for $\lambda_{i}^{1 / 2}$ which must be the same within a given block. For a real matrix $A$, its complex eigenvalues come in conjugate pairs and, by Theorem 3.4.5 in [41], the corresponding Jordan blocks $J_{k}(\lambda), J_{k}\left(\lambda^{*}\right)$ can be written in a real form $C_{k}(a, b)$ in a real basis $Z$, as in equation (2.1). The conditions for the reality of $\sqrt{A}$ are stated in subsection 2.3.1. 
Primary, nonprimary and principal square roots. A primary square root is specified by the condition that, if the eigenvalues appearing in two or more Jordan blocks of $A$ are equal, then in $F(A)$ the same branch must be chosen for all such blocks. For the remaining blocks with unequal eigenvalues, the choice of branch is unrestricted leading to multiple primary square roots. All these are expressible as polynomials in the matrix $A$ and have useful properties, in particular, under a similarity transformation $Q$, they satisfy,

$$
F\left(Q^{-1} A Q\right)=Q^{-1} F(A) Q
$$

For other functions $F(x)$ with multiple branches, the corresponding primary matrix functions $F(A)$ are defined in a similar way.

If the matrix $A$ has no eigenvalues on the negative real axis $\mathbb{R}^{-}$, then among its primary square roots there is a unique one with all eigenvalues in the open right half-plane, corresponding to choosing the principal branch for all blocks $F\left(J_{k}\right)$. This is the principal square root of $A$ which is real when $A$ is real.

Nonprimary square roots arise when $A$ has equal eigenvalues that appear in different Jordan blocks, and we do not choose the same branch for the corresponding $F\left(J_{k}\right)$. Then $A$ will have an infinite number of nonprimary square roots. An important feature of nonprimary matrix functions is that do not obey equation (A.4).

If a matrix $A$ has negative eigenvalues, then it cannot have any real primary square root, though it could have nonreal primary or real nonprimary real roots. Furthermore, a principal square root cannot be nonprimary.

A simple instructive example is the $2 \times 2$ identity matrix $A=I_{2}$ with two equal eigenvalues +1 . The primary square roots are $\pm I_{2}$ and satisfy (A.4), with $+I_{2}$ being the principal square root. A nonprimary square root is $\operatorname{diag}(+1,-1)$ which clearly violates (A.4). In fact, $Q^{-1} \operatorname{diag}(+1,-1) Q$ generates an infinite number of nonprimary square roots of $I_{2}$.

\section{B Types of blocks in the theorem}

In this section, we scrutinize all the allowed configurations and explicitly construct a common timelike vector and a common spacelike surface element relative to both metrics (exhibiting the causally coupled case), or alternatively show that such construction is not possible (resulting in two common null hyperplanes, i.e., exhibiting the null coupled case). This finalizes the sufficiency proof of the theorem. In the process, we also calculate the explicit form of a congruence that diagonalizes the metric of the geometric mean, $h=g S$.

Let us denote $Z^{\top} f Z, Z^{\top} g Z$ and $Z^{\top} h Z$ simply as $f, g$ and $h$. The assumed parameter space is $\lambda, \lambda_{k}>0, a \in \mathbb{R}, b \neq 0 \in \mathbb{R}$. Here, it is enouth to work in $2+1$ dimensions where all configurations of interest are realized, and where the null-cones can be easily visualized. The components of vectors in a tangent space (of which the null cones are subsets) will be denoted by $t \equiv x^{0}, x \equiv x^{1}$ and $y \equiv x^{2}$. In the following, the quadratic form $h=g S$ will be diagonalized by an additional congruence $X$, so that the common timelike vector for $f$, $g$ and $h$ is always set to $\tau=X \cdot(1,0,0)^{\top}$ while the common spacelike surface element to $\Sigma=X \cdot(0, x, y)^{\top}$. 
Type I. This is the case where $g^{-1} f$ could be fully diagonalized, and,

$$
g=\operatorname{diag}(-1,1,1), \quad f=\operatorname{diag}\left(-\lambda_{0}, \lambda_{1}, \lambda_{2}\right), \quad h=\operatorname{diag}\left(-\lambda_{0}^{1 / 2}, \lambda_{1}^{1 / 2}, \lambda_{2}^{1 / 2}\right) .
$$

Here, the null cones of $f$ and $g$ are centered (without the shifts) and may or may not intersect (depending on the combinations of $\lambda_{k} \gtrless 1$ ). Obviously, a congruence $X$ which diagonalizes $h$ is identity, and,

$$
\begin{array}{rlrl}
\tau^{\top} g \tau & =-1<0, & & \Sigma^{\top} g \Sigma=x^{2}+y^{2}>0, \\
\tau^{\top} f \tau=-\lambda_{0}<0, & & \Sigma^{\top} f \Sigma=\lambda_{1} x^{2}+\lambda_{2} y^{2}>0, \\
\tau^{\top} h \tau=-\lambda_{0}^{1 / 2}<0, & \Sigma^{\top} f \Sigma=\lambda_{1}^{1 / 2} x^{2}+\lambda_{2}^{1 / 2} y^{2}>0 .
\end{array}
$$

Type IIa. The diagonalization of $g^{-1} f$ is suppressed by the presence of a common tangent plane for the null cones of $f$ and $g$. In this case, $g$ is given in null coordinates,

$$
g=\operatorname{diag}\left(\epsilon\left(\begin{array}{ll}
0 & 1 \\
1 & 0
\end{array}\right), 1\right), f=\operatorname{diag}\left(\epsilon\left(\begin{array}{ll}
0 & \lambda \\
\lambda & 1
\end{array}\right), \lambda_{2}\right), h=\operatorname{diag}\left(\epsilon\left(\begin{array}{cc}
0 & \lambda^{1 / 2} \\
\lambda^{1 / 2} & \frac{1}{2} \lambda^{-1 / 2}
\end{array}\right), \lambda_{2}^{1 / 2}\right) .
$$

where $\epsilon= \pm 1$. A congruence $X$ which diagonalizes $h$ reads,

$$
X=\frac{1}{\sqrt{2}}\left(\begin{array}{cc}
-\epsilon(1+\epsilon p)^{1 / 2} & \epsilon(1-\epsilon p)^{1 / 2} \\
(1-\epsilon p)^{1 / 2} & (1+\epsilon p)^{1 / 2}
\end{array}\right), \quad p \equiv\left(1+16 \lambda^{2}\right)^{-1 / 2},
$$

where $0<p<1$ for $\lambda>0$. Consequently,

$$
\begin{aligned}
\tau^{\top} g \tau & =-\left(1-p^{2}\right)^{1 / 2}<0, & \Sigma^{\top} g \Sigma & =\left(1-p^{2}\right)^{1 / 2} x^{2}+y^{2}>0, \\
\tau^{\top} f \tau & =-\frac{(1-\epsilon p)^{2}}{4 p}<0, & \Sigma^{\top} f \Sigma & =\frac{(1+\epsilon p)^{2}}{4 p} x^{2}+\lambda_{2} y^{2}>0, \\
\tau^{\top} h \tau & =-\frac{1-\epsilon p}{2 p^{1 / 2}\left(1-p^{2}\right)^{1 / 4}}<0, & \Sigma^{\top} h \Sigma & =\frac{1+\epsilon p}{2 p^{1 / 2}\left(1-p^{2}\right)^{1 / 4}} x^{2}+\lambda_{2}^{1 / 2} y^{2}>0 .
\end{aligned}
$$

Type IIb. The null cone of $f$ is rotated and dilated by $a, b$, where we can parameterize $a=\lambda \cos \theta, b=\lambda \sin \theta$ with $\lambda=\sqrt{a^{2}+b^{2}}>0$ and $\theta \neq 0 \in(-\pi, \pi)$. (For Type IIb, necessarily $b \neq 0$, otherwise we have Type I or Type IV.) Then,

$$
\begin{aligned}
& g=\operatorname{diag}\left(\left(\begin{array}{ll}
0 & 1 \\
1 & 0
\end{array}\right), 1\right), \quad f=\operatorname{diag}\left(\left(\begin{array}{cc}
\lambda \sin \theta & \lambda \cos \theta \\
\lambda \cos \theta & -\lambda \sin \theta
\end{array}\right), \lambda_{2}\right), \\
& h=\operatorname{diag}\left(\lambda^{1 / 2}\left(\begin{array}{cc}
\operatorname{sgn}(\cos \varphi) \sin \varphi & |\cos \varphi| \\
|\cos \varphi| & -\operatorname{sgn}(\cos \varphi) \sin \varphi
\end{array}\right), \lambda_{2}^{1 / 2}\right), \quad \varphi \equiv \frac{\theta}{2} .
\end{aligned}
$$

A congruence $X$ which diagonalizes $h$ reads,

$$
X=\left(\begin{array}{ccc}
-\frac{1}{\sqrt{2}} \cos \varphi(1+\sin \varphi)^{-1 / 2} & \frac{1}{\sqrt{2}} \cos \varphi(1-\sin \varphi)^{-1 / 2} & 0 \\
\frac{1}{\sqrt{2}}(1+\sin \varphi)^{1 / 2} & \frac{1}{\sqrt{2}}(1-\sin \varphi)^{1 / 2} & 0 \\
0 & 0 & 1
\end{array}\right),
$$

where $\cos \varphi>0$ for the principal branch. Therefore,

$$
\begin{aligned}
\tau^{\top} g \tau=-\cos \varphi<0, & & \Sigma^{\top} g \Sigma=\cos \varphi x^{2}+y^{2}>0, \\
\tau^{\top} f \tau=-\lambda \cos \varphi<0, & & \Sigma^{\top} f \Sigma=\lambda \cos \varphi x^{2}+\lambda_{2} y^{2}>0, \\
\tau^{\top} h \tau=-\lambda^{1 / 2}<0, & & \Sigma^{\top} h \Sigma=\lambda^{1 / 2} x^{2}+\lambda_{2}^{1 / 2} y^{2}>0 .
\end{aligned}
$$


Type III. This is the case where the diagonalization is suppressed by the presence of a common 'saddle' tangent plane for the null cones of $f$ and $g$. Here, $g$ is given in the null coordinates of $t, y$,

$$
g=\left(\begin{array}{lll}
0 & 0 & 1 \\
0 & 1 & 0 \\
1 & 0 & 0
\end{array}\right), \quad f=\left(\begin{array}{ccc}
0 & 0 & \lambda \\
0 & \lambda & 1 \\
\lambda & 1 & 0
\end{array}\right), \quad h=\left(\begin{array}{ccc}
0 & 0 & \lambda^{1 / 2} \\
0 & \lambda^{1 / 2} & \frac{1}{2} \lambda^{-1 / 2} \\
\lambda^{1 / 2} & \frac{1}{2} \lambda^{-1 / 2} & \frac{-1}{8} \lambda^{-3 / 2}
\end{array}\right)
$$

A congruence $X$ which diagonalizes $h$ reads,

$$
X=\lambda^{-1 / 4}\left(\begin{array}{ccc}
\frac{\sqrt{2}}{4} \lambda^{-1} & 0 & \frac{\sqrt{2}}{2} \lambda^{-1} \\
\frac{\sqrt{2}}{3} & 1 & -\frac{\sqrt{2}}{3} \\
-\frac{2 \sqrt{2}}{3} \lambda & 0 & \frac{2 \sqrt{2}}{3} \lambda
\end{array}\right)
$$

This gives,

$$
\begin{aligned}
\tau^{\top} g \tau & =-\frac{4}{9} \lambda^{-1 / 2}<0, & \Sigma^{\top} g \Sigma & =\frac{1}{9} \lambda^{-1 / 2}\left((3 x-\sqrt{2} y)^{2}+12 y^{2}\right)>0, \\
\tau^{\top} f \tau & =-\frac{4}{3} \lambda^{1 / 2}<0, & \Sigma^{\top} f \Sigma & =\frac{1}{3} \lambda^{1 / 2}\left(2 x^{2}+(x+\sqrt{2} y)^{2}\right)>0, \\
\tau^{\top} h \tau & =-1<0, & \Sigma^{\top} h \Sigma & =x^{2}+y^{2}>0 .
\end{aligned}
$$

Type IV. This is a special case where,

$$
g=\operatorname{diag}(-1,1,1), \quad f=\operatorname{diag}\left(\lambda,-\lambda, \lambda_{2}\right) \quad h=\operatorname{diag}\left(\lambda^{1 / 2}\left(\begin{array}{rr}
0 & -1 \\
1 & 0
\end{array}\right), \lambda_{2}^{1 / 2}\right) .
$$

The relation $-t^{2}+x^{2}=-\left(t^{2}-x^{2}\right)$ implies that the interior one null cone is in the exterior of the other, which does not allow the existence of a common timelike vector nor a common spacelike surface element. The intersection of the cones is set of null vectors $\tau=(t, \pm t, 0)$. The two common surface elements are null hyperplanes $\Sigma_{1}=(-x, x, y)$ and $\Sigma_{2}=(x,-x, y)$.

\section{Proof of the necessary condition}

In this section, we show that if $g_{\mu \nu}$ and $f_{\mu \nu}$ are causally coupled or null coupled, then the matrix $g^{-1} f$ has a real square root. For the null coupled case, the existence of two null hyperplanes makes possible to write the metrics in the form (B.21), which is obviously sufficient for the existence of a real square root.

On the other hand, for the causally coupled case, since there exists a common spacelike hypersurface, we can find a coordinate frame where we have the simultaneous proper $3+1$ decomposition of both the metrics $g$ and $f$, given by (in matrix notation),

$$
g=\left(\begin{array}{cc}
-N^{2}+\nu^{\top} \tilde{g} \nu & \nu^{\top} \tilde{g} \\
\tilde{g} \nu & \tilde{g}
\end{array}\right), \quad f=\left(\begin{array}{cc}
-M^{2}+\mu^{\top} \tilde{f} \mu & \mu^{\top} \tilde{f} \\
\tilde{f} \mu & \tilde{f}
\end{array}\right) .
$$

Here, $N$ and $M$ are the lapses, $\nu$ and $\mu$ are the shifts, and $\tilde{g}$ and $\tilde{f}$ are the spatial restrictions of $g$ and $f$, respectively. Now, let $X=(t, x)^{\top}$ be a nonzero vector in the tangent space which belongs to the null cone of the metric $g$ that is defined by $X^{\top} g X=0$, or equivalently,

$$
(\nu+x / t)^{\top} \tilde{g}(\nu+x / t)=N^{2} .
$$


Note that $x$ and $\nu$ are spatial vectors. Since $\tilde{g}$ is positive definite, we can always find (by Cholesky decomposition) a lower-triangular $e$ (a spatial vielbein) such that $\tilde{g}=e^{\top} \tilde{\delta} e$ where $\tilde{\delta}$ denotes the spatial Euclidean metric of the local Lorentz frame. In terms of $e$, (C.2) can be written in a more symmetric form,

$$
\left(N^{-1} e(\nu+x / t)\right)^{\top} \tilde{\delta}\left(N^{-1} e(\nu+x / t)\right)=\alpha^{2},
$$

where we introduced a real parameter $\alpha$ for which $\alpha^{2}=1$ defines the null cone, while $\alpha^{2}<1$ defines its interior. By further introducing a local Lorentz frame spatial vector $u$,

$$
u \equiv N^{-1} e(\nu+x / t)
$$

we can write (C.3) as $u^{\top} \tilde{\delta} u=\alpha^{2}$, and parameterize the null cone as the set of all null rays,

$$
x=\left(N e^{-1} u-\nu\right) t, \quad t \in \mathbb{R} \backslash\{0\},
$$

that is generated by vectors $u$ on a unit sphere $|u|^{2} \equiv u^{\top} \tilde{\delta} u=u^{a} \delta_{a b} u^{b}=\alpha^{2}=1$. In such case, the open set (a soft ball) $\alpha^{2}<1$ correspondingly defines the null cone interior. $^{18}$ The local vector $u$ is, of course, determined up to an $\mathrm{O}(3)$ rotation that keeps the spatial Euclidean metric $\tilde{\delta}$ invariant, i.e., $\tilde{\delta}=R^{\top} \tilde{\delta} R$. Geometrically, the spatial metric $\tilde{g}$ (or the corresponding $e$ ) will deform a $S^{2}$-sphere $|u|^{2}=1$ ("the light front" at fixed time) into an ellipsoid, further scaled by the lapse $N$ (the local passage of time) which will finally be centered at the shift $-\nu$ (that corresponds to shear of time relative to the spacelike hypersurface).

Now, let us parameterize the interiors of null cones $g$ and $f$ by $u_{1}$ and $u_{2}$, respectively. Then, if the interiors of the null cones intersect, there exists some $\alpha$ for which we can find a common $u=-R u_{1}=u_{2}$ (up to a spatial rotation $R$ ) so that,

$$
x / t=N e^{-1} u-\nu=-M(R m)^{-1} u-\mu,
$$

where $u^{\top} \tilde{\delta} u=\alpha^{2}<1$. This yields,

$$
\nu-\mu=\left(N e^{-1}+M(R m)^{-1}\right) u,
$$

which is the sufficient condition for the existence of the real square root [46].

\section{Geometric mean of symmetric matrices}

Consider a matrix function $F\left(g^{-1} f\right)$ for an arbitrary scalar stem function $F(X)$, and also define,

$$
h_{F} \equiv g F\left(g^{-1} f\right) \text {. }
$$

Accordingly,

$$
Z^{\top} h_{F} Z=\operatorname{diag}\left(\epsilon_{1} E_{1} F\left(J_{1}\right), \ldots, \epsilon_{q} E_{q} F\left(J_{q}\right), F\left(C_{q+1}\right), \ldots, F\left(C_{p}\right)\right) .
$$

\footnotetext{
${ }^{18}$ One can also fix $|u|^{2}=1$ and change $N \rightarrow \alpha N$.
} 
The upper triangular strip form in (A.2) implies that $h_{F}$ in (D.2) is symmetric, $h_{F}=h_{F}^{\top}$, for any stem function $F .{ }^{19}$ Hence, a covariant tensor associated with $h_{F}$ can function as a metric. When $F$ is on the principal branch, $h_{F}$ has the same signature as $f$ and $g$.

Now, take the specific stem function,

$$
F(X)=X^{\xi} \equiv \exp (\xi \log X),
$$

for a real $\xi$. For this stem, we can define the composite metric,

$$
g_{\xi} \equiv g\left(g^{-1} f\right)^{\xi}
$$

where, in particular: $g_{0}=g, g_{1}=f$, and for $\xi=1 / 2$ (taking the principal square root),

$$
h \equiv g_{1 / 2}=g\left(g^{-1} f\right)^{1 / 2}=f\left(f^{-1} g\right)^{1 / 2}=f \# g=g \# f .
$$

Here, we used $A \# B$ to denote the geometric mean of $A$ and $B$ (section 2.4 in [33]). For the null cones, the term geometric mean is well justified, since one has,

$$
H^{2}=\gamma^{-1}\left(M^{2} N^{2}\right)^{1 / 2}, \quad \operatorname{det} \tilde{h}=\gamma(\operatorname{det} \tilde{f} \operatorname{det} \tilde{g})^{1 / 2},
$$

in terms of the spatial parts $\tilde{f}, \tilde{g}, \tilde{h}$ and the lapses $M, N$ and $H$ of $f, g$ and $h$, respectively, where the $\gamma$-factor ranges $0<\gamma^{-1}=\sqrt{x} \leq 1,[46]$.

It is easy to verify that the set of all $g_{\xi}$ stays closed under the binary operation,

$$
g_{\xi}=g_{\xi_{1}} \# g_{\xi_{2}}=g_{\xi_{1}}\left(g_{\xi_{1}}^{-1} g_{\xi_{2}}\right)^{1 / 2}=g\left(g^{-1} f\right)^{\left(\xi_{1}+\xi_{2}\right) / 2}=g_{\left(\xi_{1}+\xi_{2}\right) / 2},
$$

especially on the segment $\xi, \xi_{1}, \xi_{2} \in[0,1]$. This makes possible the ordering $g_{\xi_{1}} \prec g_{\xi_{2}} \Leftrightarrow$ $\xi_{1}<\xi_{2}$, and $g=g_{0} \prec g_{\xi} \prec g_{1}=f$ for $0<\xi<1$. Finally, note that the null cones of all metrics $g_{\xi}$ are causally coupled to each other.

\section{E Absence of relative time orientation flip}

As shown in the main text, Type IV metrics are obtainable as a limit of Type IIb when $\theta \rightarrow \pi$. Although, as argued in the text, Type IV metrics cannot arise as valid solutions, on the face of it, crossing $\theta=\pi$ could lead to a problem with time orientability. Here we show that this is not the case. Geometrically, in terms of IIb null cones in figure 4, $\theta$ is the angle between the left edge of the blue cone and the right edge of the red cone. Initially, for $\theta<\pi$, the null cones have common timelike directions within their intersection. As $\theta \rightarrow \pi$, the intersection shrinks to zero, and the null cones touch along two null directions (Type IV in figure 4). One null direction is a limit of the common timelike vectors while the other null direction was initially on a spacelike hypersurface. If $\theta$ increases beyond $\pi$ the null cones intersect again, in a new IIb configuration. However, now what was the future cone of $f$ intersects with the past cone of $g$, so seemingly the two metrics develop opposite time orientations.

\footnotetext{
${ }^{19}$ Note that this holds even for nonprimary functions (see section 1.4 in [33]).
} 
This problem will not arise if the transition through $\theta=\pi$ is accompanied by a time reversal for the $f$ metric. This is not prohibited for the following reason. The new common time directions for $\theta>\pi$ are not continuations of the old timelike directions for $\theta<\pi$. Rather, they arise from the Type IV null direction that originated from spacelike directions for $\theta<\pi$. Similarly, the spatial hypersurfaces for $\theta>\pi$ arise from the Type IV null direction that was initially timelike (In principle, a $2+2$ decomposition can describe such a transition through $\theta=\pi$ ). This lack of continuity in the common time directions allows for a reinterpretation of time orientation. Below we show that specifying a branch for the square root matrix indeed implements this mechanism, ensuring that the time orientations of $f$ and $g$ remain compatible.

When a Type IIb configuration crosses $\theta=\pi$ into a new Type IIb configuration, one encounters a potential problem with the relative time-orientability of the two metrics, as discussed above. We now show that the transition through $\theta=\pi$ is accompanied by time and space reflections of the $f$ metric (in our parametrization) so that the relative time orientations of the two metrics remain unchanged. This can be seen directly by noting that while the $f$ metric of Type IIb (as parametrized in (2.12)) is continuous at $\theta=\pi$, its vielbein is not and exhibits the time and space reflections. The $f$ vielbein can be easily obtained as follows. Associated with a complex number $z$, consider the matrix,

$$
\mathcal{C}(z) \equiv\left(\begin{array}{rr}
\operatorname{Re} z & -\operatorname{Im} z \\
\operatorname{Im} z & \operatorname{Re} z
\end{array}\right)
$$

where $\mathcal{C}(1)$ is the identity matrix. Then the following equations hold,

$$
\mathcal{C}(z) \mathcal{C}(w)=\mathcal{C}(z w), \quad \mathcal{C}(z)^{-1}=\mathcal{C}\left(z^{-1}\right), \quad \mathcal{C}(z)^{\top}=\mathcal{C}(\bar{z}), \quad \sqrt{\mathcal{C}(z)}=\mathcal{C}(\sqrt{z})
$$

where the last equation follows from (3.9). The reversal matrix associates a metric to $z$,

$$
\hat{f}(z) \equiv\left(\begin{array}{ll}
0 & 1 \\
1 & 0
\end{array}\right) \mathcal{C}(z), \quad \hat{f}(z)=\hat{f}(1) \mathcal{C}(z), \quad \hat{f}(z)=\hat{f}^{\top}(z)
$$

Note that $\hat{f}(1) \equiv \hat{\eta}$ is the $2 \times 2$ Minkowski metric in the null frame and $\hat{f}(z)$ is the $2 \times 2$ block of the metric $f$ in $(2.12)$. Since $\mathcal{C}(z)=\mathcal{C}(\sqrt{z}) \mathcal{C}(\sqrt{z})$ and $\hat{f}(1) \mathcal{C}(\sqrt{z})$ is symmetric, we can write,

$$
\hat{f}=\mathcal{C}(\sqrt{z})^{\top} \hat{f}(1) \mathcal{C}(\sqrt{z})
$$

Hence, up to local Lorentz transformations, the vielbein of $\hat{f}=\hat{L}^{\top} \hat{\eta} \hat{L}$ is given by

$$
\hat{L}=\mathcal{C}(\sqrt{z}) \text {. }
$$

But $\mathcal{C}(\sqrt{z})$ is the square root matrix (3.9) which is discontinuous at $\theta=\pi$. Then, from (3.7) and (3.8) it is obvious that,

$$
\lim _{\theta \rightarrow \pi^{-}} \hat{L}=\left(\begin{array}{rr}
0 & -1 \\
1 & 0
\end{array}\right), \quad \lim _{\theta \rightarrow \pi^{+}} \hat{L}=-\left(\begin{array}{rr}
0 & -1 \\
1 & 0
\end{array}\right) .
$$

The sign change across $\theta=\pi$ is due to a reflection in the time and in a space direction and ensures that the future cones of $f$ and $g$ continue intersecting. Embedding this setup in four dimensions and taking the congruence $Z$ into account replaces the $f$ vielbein by $L Z$ but does not change the above conclusions. 
Open Access. This article is distributed under the terms of the Creative Commons Attribution License (CC-BY 4.0), which permits any use, distribution and reproduction in any medium, provided the original author(s) and source are credited.

\section{References}

[1] M. Fierz and W. Pauli, On relativistic wave equations for particles of arbitrary spin in an electromagnetic field, Proc. Roy. Soc. Lond. A 173 (1939) 211.

[2] D.G. Boulware and S. Deser, Can gravitation have a finite range?, Phys. Rev. D 6 (1972) 3368 [INSPIRE].

[3] C.J. Isham, A. Salam and J.A. Strathdee, F-dominance of gravity, Phys. Rev. D 3 (1971) 867 [INSPIRE].

[4] B. Zumino, Effective Lagrangians and Broken Symmetries, in Lectures on Elementary Particles and Quantum Field Theory, volume 2, Brandeis University, Cambridge, MA, U.S.A., (1970), pp. 437-500.

[5] N. Boulanger, T. Damour, L. Gualtieri and M. Henneaux, Inconsistency of interacting, multigraviton theories, Nucl. Phys. B 597 (2001) 127 [hep-th/0007220] [INSPIRE].

[6] C. de Rham, G. Gabadadze and A.J. Tolley, Resummation of Massive Gravity, Phys. Rev. Lett. 106 (2011) 231101 [arXiv: 1011.1232] [INSPIRE].

[7] S.F. Hassan and R.A. Rosen, Resolving the Ghost Problem in non-Linear Massive Gravity, Phys. Rev. Lett. 108 (2012) 041101 [arXiv:1106.3344] [INSPIRE].

[8] S.F. Hassan and R.A. Rosen, Bimetric Gravity from Ghost-free Massive Gravity, JHEP 02 (2012) 126 [arXiv:1109.3515] [INSPIRE].

[9] S.F. Hassan and R.A. Rosen, Confirmation of the Secondary Constraint and Absence of Ghost in Massive Gravity and Bimetric Gravity, JHEP 04 (2012) 123 [arXiv:1111.2070] [INSPIRE].

[10] N. Arkani-Hamed, H. Georgi and M.D. Schwartz, Effective field theory for massive gravitons and gravity in theory space, Annals Phys. 305 (2003) 96 [hep-th/0210184] [INSPIRE].

[11] P. Creminelli, A. Nicolis, M. Papucci and E. Trincherini, Ghosts in massive gravity, JHEP 09 (2005) 003 [hep-th/0505147] [INSPIRE].

[12] M. Ostrogradsky, Mémoires sur les équations différentielles, relatives au problème des isopérimètres, (In French), Mem. Ac. St. Petersbourg 4 (1850) 385.

[13] C. de Rham and G. Gabadadze, Generalization of the Fierz-Pauli Action, Phys. Rev. D 82 (2010) 044020 [arXiv: 1007.0443] [INSPIRE].

[14] S.F. Hassan and R.A. Rosen, On Non-Linear Actions for Massive Gravity, JHEP 07 (2011) 009 [arXiv:1103.6055] [INSPIRE].

[15] S.F. Hassan, R.A. Rosen and A. Schmidt-May, Ghost-free Massive Gravity with a General Reference Metric, JHEP 02 (2012) 026 [arXiv:1109.3230] [INSPIRE].

[16] S.F. Hassan, A. Schmidt-May and M. von Strauss, Proof of Consistency of Nonlinear Massive Gravity in the Stúckelberg Formulation, Phys. Lett. B 715 (2012) 335 [arXiv: 1203.5283] [INSPIRE]. 
[17] D. Comelli, M. Crisostomi, F. Nesti and L. Pilo, Degrees of Freedom in Massive Gravity, Phys. Rev. D 86 (2012) 101502 [arXiv:1204.1027] [INSPIRE].

[18] J. Kluson, Non-Linear Massive Gravity with Additional Primary Constraint and Absence of Ghosts, Phys. Rev. D 86 (2012) 044024 [arXiv:1204.2957] [InSPIRE].

[19] C. Deffayet, J. Mourad and G. Zahariade, Covariant constraints in ghost free massive gravity, JCAP 01 (2013) 032 [arXiv:1207.6338] [INSPIRE].

[20] T. Kugo and N. Ohta, Covariant Approach to the No-ghost Theorem in Massive Gravity, PTEP 2014 (2014) 043B04 [arXiv: 1401.3873] [INSPIRE].

[21] M. Kocic, Geometric mean of bimetric spacetimes, arXiv:1803.09752 [INSPIRE].

[22] S.F. Hassan, A. Schmidt-May and M. von Strauss, On Consistent Theories of Massive Spin-2 Fields Coupled to Gravity, JHEP 05 (2013) 086 [arXiv:1208.1515] [INSPIRE].

[23] C. de Rham, Massive Gravity, Living Rev. Rel. 17 (2014) 7 [arXiv:1401.4173] [INSPIRE].

[24] A. Schmidt-May and M. von Strauss, Recent developments in bimetric theory, J. Phys. A 49 (2016) 183001 [arXiv:1512.00021] [INSPIRE].

[25] P. Martín-Moruno, V. Baccetti and M. Visser, Massive gravity as a limit of bimetric gravity, in Proceedings, 13th Marcel Grossmann Meeting on Recent Developments in Theoretical and Experimental General Relativity, Astrophysics and Relativistic Field Theories (MG13): Stockholm, Sweden, July 1-7, 2012, pp. 1270-1272, arXiv:1302.2687 [INSPIRE].

[26] Y. Akrami, S.F. Hassan, F. Könnig, A. Schmidt-May and A.R. Solomon, Bimetric gravity is cosmologically viable, Phys. Lett. B 748 (2015) 37 [arXiv:1503.07521] [INSPIRE].

[27] S.F. Hassan, A. Schmidt-May and M. von Strauss, Extended Weyl Invariance in a Bimetric Model and Partial Masslessness, Class. Quant. Grav. 33 (2016) 015011 [arXiv:1507.06540] [INSPIRE].

[28] E. Gourgoulhon, 3+1 Formalism in General Relativity, Springer (2012), [https://doi.org/10.1007/978-3-642-24525-1].

[29] Y. Choquet-Bruhat and R.P. Geroch, Global aspects of the Cauchy problem in general relativity, Commun. Math. Phys. 14 (1969) 329 [INSPIRE].

[30] A.N. Bernal and M. Sanchez, On smooth Cauchy hypersurfaces and Geroch's splitting theorem, Commun. Math. Phys. 243 (2003) 461 [gr-qc/0306108] [INSPIRE].

[31] P.A.M. Dirac, The Theory of gravitation in Hamiltonian form, Proc. Roy. Soc. Lond. A 246 (1958) 333.

[32] R.L. Arnowitt, S. Deser and C.W. Misner, Canonical variables for general relativity, Phys. Rev. 117 (1960) 1595 [INSPIRE].

[33] N.J. Higham, Functions of Matrices: Theory and Computation, SIAM, (2008), [https://doi.org/10.1137/1.9780898717778].

[34] N.J. Higham, Computing real square roots of a real matrix, Linear Algebra Appl. 88-89 (1987) 405.

[35] R.A. Horn and C.R. Johnson. Topics in Matrix Analysis, Cambridge University Press, (1994), [https://doi.org/10.1017/CBO9780511840371].

[36] F. Uhlig, Simultaneous block diagonalization of two real symmetric matrices, Linear Algebra Appl. 7 (1973) 281. 
[37] F. Uhlig, A canonical form for a pair of real symmetric matrices that generate a nonsingular pencil, Linear Algebra Appl. 14 (1976) 189.

[38] F. Uhlig, A recurring theorem about pairs of quadratic forms and extensions: a survey, Linear Algebra Appl. 25 (1979) 219.

[39] F.R. Gantmacher, The Theory of Matrices, volume 2, Chelsea (1959).

[40] Y.P. Hong, R.A. Horn and C.R. Johnson, On the reduction of pairs of Hermitian or symmetric matrices to diagonal form by congruence, Linear Algebra Appl. 73 (1986) 213.

[41] R.A. Horn and C.R. Johnson, Matrix Analysis, Cambridge University Press, (1990), [https://doi.org/10.1017/CBO9781139020411].

[42] V. Baccetti, P. Martín-Moruno and M. Visser, Gordon and Kerr-Schild ansatze in massive and bimetric gravity, JHEP 08 (2012) 108 [arXiv: 1206.4720] [INSPIRE].

[43] S. Dey, A. Fring and T. Mathanaranjan, Spontaneous PT-Symmetry Breaking for Systems of Noncommutative Euclidean Lie Algebraic Type, Int. J. Theor. Phys. 54 (2015) 4027 [INSPIRE].

[44] R.A. d'Inverno and J. Smallwood, Covariant 2+2 formulation of the initial-value problem in general relativity, Phys. Rev. D 22 (1980) 1233 [INSPIRE].

[45] S.F. Hassan, A. Schmidt-May and M. von Strauss, Bimetric theory and partial masslessness with Lanczos-Lovelock terms in arbitrary dimensions, Class. Quant. Grav. 30 (2013) 184010 [arXiv:1212.4525] [INSPIRE].

[46] S.F. Hassan, M. Kocic and A. Schmidt-May, Absence of ghost in a new bimetric-matter coupling, arXiv:1409.1909 [INSPIRE].

[47] L. Bernard, C. Deffayet and M. von Strauss, Massive graviton on arbitrary background: derivation, syzygies, applications, JCAP 06 (2015) 038 [arXiv:1504.04382] [INSPIRE].

[48] K. Izumi and Y.C. Ong, An analysis of characteristics in nonlinear massive gravity, Class. Quant. Grav. 30 (2013) 184008 [arXiv: 1304.0211] [INSPIRE].

[49] S. Deser, K. Izumi, Y.C. Ong and A. Waldron, Massive Gravity Acausality Redux, Phys. Lett. B 726 (2013) 544 [arXiv: 1306.5457] [INSPIRE].

[50] S. Deser, K. Izumi, Y.C. Ong and A. Waldron, Superluminal Propagation and Acausality of Nonlinear Massive Gravity, in Proceedings, Conference in Honor of the 90th Birthday of Freeman Dyson: Singapore, Singapore, August 26-29, 2013, pp. 430-435, 2014, arXiv:1312.1115 [INSPIRE].

[51] S. Deser, M. Sandora, A. Waldron and G. Zahariade, Covariant constraints for generic massive gravity and analysis of its characteristics, Phys. Rev. D 90 (2014) 104043 [arXiv: 1408.0561] [INSPIRE].

[52] S. Deser, K. Izumi, Y.C. Ong and A. Waldron, Problems of massive gravities, Mod. Phys. Lett. A 30 (2015) 1540006 [arXiv:1410.2289] [InSPIRE].

[53] R. Geroch, Faster Than Light?, AMS/IP Stud. Adv. Math. 49 (2011) 59 [arXiv:1005.1614] [INSPIRE].

[54] E. Babichev, V. Mukhanov and A. Vikman, $k$-Essence, superluminal propagation, causality and emergent geometry, JHEP 02 (2008) 101 [arXiv:0708.0561] [INSPIRE].

[55] M. Düll, F.P. Schuller, N. Stritzelberger and F. Wolz, Gravitational closure of matter field equations, Phys. Rev. D 97 (2018) 084036 [arXiv:1611.08878] [INSPIRE]. 
[56] I.T. Drummond, Quantum field theory in a multimetric background, Phys. Rev. D 88 (2013) 025009 [arXiv: 1303.3126] [INSPIRE].

[57] M.S. Volkov, Stability of Minkowski space in ghost-free massive gravity theory, Phys. Rev. D 90 (2014) 024028 [arXiv: 1402.2953] [INSPIRE].

[58] M.S. Volkov, Energy in ghost-free massive gravity theory, Phys. Rev. D 90 (2014) 124090 [arXiv: 1404.2291] [INSPIRE].

[59] S.F. Hassan, A. Schmidt-May and M. von Strauss, Particular Solutions in Bimetric Theory and Their Implications, Int. J. Mod. Phys. D 23 (2014) 1443002 [arXiv:1407.2772] [INSPIRE].

[60] K. Hinterbichler and R.A. Rosen, Interacting Spin-2 Fields, JHEP 07 (2012) 047 [arXiv: 1203.5783] [INSPIRE].

[61] M. Bojowald, Canonical Gravity and Applications, Cambridge University Press, (2010), [https://doi.org/10.1017/CBO9780511921759].

[62] X.O. Camanho, G. Lucena Gómez and R. Rahman, Causality Constraints on Massive Gravity, Phys. Rev. D 96 (2017) 084007 [arXiv: 1610.02033] [InSPIRE].

[63] S. Alexandrov, Canonical structure of Tetrad Bimetric Gravity, Gen. Rel. Grav. 46 (2014) 1639 [arXiv: 1308.6586] [INSPIRE].

[64] S.F. Hassan and A. Lundkvist, Analysis of constraints and their algebra in bimetric theory, arXiv: 1802.07267 [INSPIRE].

[65] L. Bernard, C. Deffayet, A. Schmidt-May and M. von Strauss, Linear spin-2 fields in most general backgrounds, Phys. Rev. D 93 (2016) 084020 [arXiv:1512.03620] [InSPIRE].

[66] C. Deffayet, J. Mourad and G. Zahariade, A note on 'symmetric' vielbeins in bimetric, massive, perturbative and non perturbative gravities, JHEP 03 (2013) 086 [arXiv: 1208.4493] [INSPIRE].

[67] S.F. Hassan, A. Schmidt-May and M. von Strauss, Metric Formulation of Ghost-Free Multivielbein Theory, arXiv:1204.5202 [INSPIRE].

[68] L. Apolo and S.F. Hassan, Non-linear partially massless symmetry in an $\mathrm{SO}(1,5)$ continuation of conformal gravity, Class. Quant. Grav. 34 (2017) 105005 [arXiv: 1609.09514] [INSPIRE].

[69] C. Cheung and G.N. Remmen, Positive Signs in Massive Gravity, JHEP 04 (2016) 002 [arXiv: 1601.04068] [INSPIRE].

[70] D. Comelli, M. Crisostomi, K. Koyama, L. Pilo and G. Tasinato, New Branches of Massive Gravity, Phys. Rev. D 91 (2015) 121502 [arXiv:1505.00632] [InSPIRE]. 